\title{
Die Entstehung des periphrastischen Perfekts mit haben und sein im Deutschen - eine längst beantwortete Frage?
}

\author{
PETER ÖHL
}

\section{Abstract}

The rise of the periphrastic perfect tenses - past participle + have or be is a phenomenon for which the analysis is especially promising with regards to the research on grammaticalisation. Firstly, it involved several steps of syntactic reanalysis and lexical recategorisation leading to a constructionspecific auxiliary verb form. This allows for conclusions to be drawn about processes involved in grammaticalisation in general. Secondly, it is a grammatical change in which both Germanic and Romance languages converged. This allows for conclusions about universal conditions underlying such kinds of change. Thirdly, in German, the rise occurred rather late and within a relatively short period of time. This allows for the observation of the periphrasis in statu nascendi. In this paper, the grammaticalisation process is modeled and explained in a formal framework of generative syntax, also taking into account performance-based changes and contextual aspects providing the conditions for the relevant changes in the grammar. The main claim is that this process of grammaticalisation was terminated by an abrupt parametric change, leading to a syntactically reanalysed form of analytic inflection and the recategorisation of have as an auxiliary. The result was a full paradigm of periphrastic perfect tense. The preconditions for this grammatical change were provided by a sequence of performancebased changes and minor formal reanalyses, giving the process its gradual nature.

Keywords: Grammaticalisation, Old High German, Latin, formal and functional accounts

\section{Einleitung: Formale und funktionale Erklärungsansätze}

für die Auxiliarisierung

Die Entstehung periphrastischer Formen in den Tempussystemen indogermanischer Sprachen, insbesondere auch des Deutschen, ist ein empi- 
risch (vgl. Oubouzar 1975) seit langem recht gut dokumentiertes Phänomen, dem auch in der gegenwärtigen Sprachwandelforschung einige Aufmerksamkeit zuteil wurde. In systematischen Untersuchungen geht es vor allem um Fragen wie die Entstehung verbaler Periphrasen aus adjektivischen oder prädikativen Kurzsatzstrukturen (vgl. z. B. Ramat 1982; Abraham 1991, 1992a) oder die Auslöser der Grammatikalisierung des Auxiliars, seine semantische Entleerung und lexikalische Rekategorisierung (Heine 1993; vgl. auch versch. Beiträge in Harris \& Ramat 1987, z. T. diskutiert in Abraham 1990).

Mehrfach und aus verschiedenen Blickwinkeln untersucht wurde u. a. das periphrastische Perfekt mit haben + Partizip II ${ }^{1}$ (fortan PII).

(1) Ich habe geschlafen.

(vgl. Behaghel 1899)

Verschiedene traditionelle Ansätze, wie z. B. Brinkmann (1931) oder Grønvik (1986), legen der Herausbildung des Perfekts im Germanischen ein Modell der Lehnsyntax zugrunde, das dessen verhältnismäßig kurze Entstehungszeit erklären helfen soll: „Das haben-Perfekt tritt im deutschen Raum plötzlich als unvorbereitete Neuerung auf. Im Romanischen hat das „haben“-Perfekt eine lange Geschichte, die sich bis zu Plautus zurückverfolgen lässt.“ (Brinkmann 1931: 25).

U.E. ist jedoch die Entstehung dieser Konstruktion im Germanischen und Romanischen durch zwar zeitversetzte, aber konvergente Grammatikalisierungs- und Reanalyseprozesse zu erklären. ${ }^{2}$ Im vorliegenden Beitrag wollen wir zunächst derartige Annahmen, dass die Konstruktion im Deutschen plötzlich und unvermittelt als Entlehnung aus dem Romanischen aufgetreten sei, durch formale, funktionale und historische Argumente widerlegen. Ebenso werden wir der Ansicht widersprechen, dass die Grammatikalisierung des habere-Perfekts bereits im klassischen Latein begonnen hätte.

$\mathrm{Zu}$ diesem Zweck werden wir mithilfe eines integrativen Sprachwandelmodells die einzelnen in die Entstehung der Konstruktion involvierten Phänomene rekonstruieren. Unser Modell berücksichtigt sowohl funktional zu erklärende, performanzbasierte Prozesse, wie auch die abrupten parametrischen Wandel, die letztlich das syntaktische Regelsystem derje-

1. Wir schließen uns der verbreiteten Ansicht an, dass in Sprachen wie dem Neuhochdeutschen Perfektpartizip und Passivpartizip nicht separat klassifiziert werden können. So argumentiert Abraham (2006: 8 f.), dass die Passivbedeutung des Partizips von der stativen Bedeutung des Perfektpartizips abzuleiten sei. Wir subsumieren beide Partizipien unter der Notation ,PII'.

2. Verschiedene weitere Argumente gegen die syntaktische Entlehnung der Konstruktion aus dem Lateinischen finden sich z. B. in Morris (1988). 
nigen Sprachen restrukturierten, die diese Art der periphrastischen Perfektkonstruktion entwickelt haben.

Interessante Aufschlüsse über die Entstehung der Perfektperiphrase gibt hierbei das Althochdeutsche (fortan Ahd.), da es hier im Vergleich zu den anderen westgermanischen Sprachen ein ,Nachzügler' war und deshalb die Perfektperiphrase in statu nascendi zu beobachten erlaubt. Die ahd. Quellen ermöglichen die chronologische Betrachtung aufeinander folgender Wandel, die u. E. in gleicher oder zumindest vergleichbarer Weise auch in den anderen germanischen Sprachen, und ebenso im Romanischen, zur Bildung des periphrastischen Perfekts geführt haben.

Unsere Annahmen sehen wir in der Distribution der Perfektauxiliare haben und sein bestätigt, die wir abschließend diskutieren. Bekanntlich dient(e) sowohl in romanischen wie auch germanischen Sprachen bei Verben bestimmten Typs nicht haben, sondern sein als Auxiliar für die Perfektkonstruktion.

a. Je suis allé á l'école

b. Ich bin zur Schule gegangen

Auch dieser Umstand ist aus der Entstehung des periphrastischen Perfekts heraus erklärbar. Wir beleuchten die Entwicklung aus dem Blickwinkel funktionalistischer (Dik 1987; Shannon 1990) wie auch generativistischer Erklärungen (Haider 1984, 2000b; Burzio 1986; Haider \& Rindler-Schjerve 1987; Abraham 2000) und schlagen hierfür ebenfalls eine integrative Erklärung vor, die die wesentlichen Elemente beider Erklärungsansätze miteinander verbindet.

\section{Datenlage und Problemstellung}

\subsection{Althochdeutsche Übersetzungsliteratur}

Die Perfektkonstruktion ist in den germanischen Sprachen erst spät entstanden. Das Bibelgotische (4. Jh. n. Chr.) kennt sie beispielsweise überhaupt nicht (vgl. Dal 1966: 121). Das Tempussystem des (rekonstruierten) Protogermanischen kam mit zwei Tempora aus: einem für Sachverhalte, die vor und bis zur Gegenwart situiert waren, dem Präteritum, ${ }^{3}$

3. Vgl. Behaghel (1924: 282): „Das einfache Präteritum bezeichnet ursprünglich (idg., urgerm., got., wgm.) jede in der Vergangenheit liegende Tatsache: einen Zustand wie einen Vorgang. Dabei ist es ganz gleichgültig, ob die Tatsache der Vergangenheit in der Gegenwart nachwirkt oder nicht." 
und einem für solche ab der Gegenwart, dem Präsens. ${ }^{4}$ In den ahd. Übersetzungen aus dem Lateinischen um 800 n. Chr. werden, wie im Bibelgotischen, dessen Übersetzungsvorlage das Griechische war, sowohl Perfekt als auch Plusquamperfekt mit synthetischem Präteritum übersetzt. In späteren deutschen Bibelversionen stehen an selber Stelle Perfekt oder Plusquamperfekt.

(3) a. usiddja unhulpo us dauhtr peinai ausging Unhold aus Tochter deiner (got.; Wulfila: Mc 7,29)

b. Der Teufel ist aus Deiner Tochter ausgefahren. (Luther)

c. Der Dämon hat deine Tochter verlassen. (Hausbibel)

a. quia super pauca fuisti fidelis, super multa weil über wenige sein-PERF-2.Sg treu über viele te constituam. dich einsetze

(Vulg: Mt 25,23)

b. uuanta thu ubar fohiu uuari gitriuni, managu thih gisezzu. weil du über wenige warst treu viele dich setze

(Tat. 149,5)

c. Du bist über wenigem getreu gewesen; ich will dich über viel setzen

d. Du bist im kleinen ein treuer Verwalter gewesen, ich will Dir eine große Aufgabe übertragen.

(Hausbibel)

(5) a. cum consummasset Ihesus verba haec als beend- $P Q P$-3. Sg Jesus Worte diese

b. mit diu gientota ther heilant thisu unort mit dem beendete der Heiland diese Worte (Tat 43,3)

c. da Jesus diese Rede vollendet hatte

(Luther)

(6)

a. abiit autem qui V talenta acceperat davongeh-PERF aber der 5 Talente 3mpfang- $P Q P-3 . S g$ et operatus est in eis et lucratus est alia und arbeit-PII $A U X$ in diesen und gewinn-PII $A U X$ andere quinque

fünf

(Vulg: Mt 25, 16)

4. Dies bedeutet jedoch nicht, dass Aspekt als verbale Kategorie nicht vorhanden war. So argumentiert Leiss (1992: 54 ff.) für eine paradigmatische morphologische Aspektmarkierung im Germanischen. Diese lebe im deutschen Präfix ge- fort, was die Lexikalisierung der Perfektivität entsprechender Verben begründen kann (z. B. gelangen, gereichen, gewinnen), aber auch die analogische Ausdehnung des Präfixes im Paradigma der PII. 
b. ging tho ther thio fimf talente infing inti uuarohta in ging da der die fünf Talente empfing und arbeitete in then inti gistriunita andero fimui denen und gewann andere fünf

c. Da ging der hin, der fünf Zentner empfangen hatte, und handelte mit denselbigen und gewann andere fünf Zentner.

d. Sofort begann der Diener, der die fünf Talente erhalten hatte, mit ihnen zu wirtschaften, und er gewann noch fünf dazu.

(Hausbibel)

Die folgenden beiden Tatianbelege zeigen aber nicht nur, dass der Übersetzer, im Gegensatz zu Martin Luther oder den Autoren der Hausbibel im 20. Jh., kein haben-Perfekt zur Verfügung hatte, um die lateinischen Perfektformen tradisti und superlucratus sum wiederzugeben. Gleichzeitig spiegeln diese Belege unterschiedliche Übersetzungsstrategien zur Wiedergabe einer Konstruktion wider, für die in der Zielsprache kein Äquivalent existierte. U.E. sind diese Stellen so zu interpretieren, dass der Übersetzer auf zwei verschiedene Weisen versuchte, mittels einer ahd. Prädikativkonstruktion der Form esse + PII im zweiten Konjunkt der lat. Vorlage nahe zu kommen, sich augenscheinlich jedoch für keine der beiden als angemessen entscheiden konnte.

a. Domine, $V$ talenta tradisti mihi, ecce alia Herr 5 Talente übergeb-PERF-2.Sg mir siehe andere quinque superlucratus sum fünf gewinnen-PII $A U X$

b. Herro, fimf talenta saltostu Herr fünf Talente geb-PR ̈̈T-2.Sg mir siehe andere haben gistriunit hab-PRÄS-1.Sg. gewinnen-PII

c. Herr, du hast mir fünf Zentner getan; siehe da, ich habe damit andere fünf Zentner gewonnen. (Luther)

d. Herr, fünf Talente hast Du mir gegeben; sieh her, ich habe noch fünf dazugewonnen.

(Hausbibel)

a. Domine, duo talenta tradisti mihi, ecce

Herr zwei Talente übergeb-PERF-2.Sg mir siehe alia duo superlucratus sum andere zwei gewinn-PII $A U X$

b. Herro, zuua talenta saltostu mir, senu andero zuиa Herr zwei Talente geb-PRÄT-2.Sg mir siehe andere zwei gistriunenti bin gewinnender bin

(Tat 149,5) 
c. Herr, du hast mir zween Zentner getan; siehe da, ich habe mit denselben zween andere gewonnen (Luther)

d. Herr, Du hast mir zwei Talente gegeben; sieh her, ich habe noch fünf dazugewonnen.

(Hausbibel)

(7b) ist keine Perfektkonstruktion, sondern ein PII in Funktion eines sekundären Prädikats unter dem Hauptverb haben. Die vorliegende Struktur war zwar Ausgangspunkt der Reanalyse des haben-Perfekts, dieses existierte aber zu diesem Zeitpunkt noch nicht (vgl. hierzu unten, 2.3; 5.2). (8b) ist die Konstruktion Kopula + Gerundium, die dem Übersetzer ermöglichte, zumindest das formale Äquivalent des lat. Auxiliars einzusetzen. Man beachte, dass er im obigen Beispiel (6b) dagegen zur Übersetzung der ähnlichen Form lucratus est das einfache Präteritum verwendet. 5

Der klarste Beleg dafür, dass das einfache Präteritum als funktionales Äquivalent von Perfekt und Plusquamperfekt galt, ist die einheitliche Wiedergabe in den ahd. Glossen (vgl. Grimm, Dt. Gramm IV: 176), z. B.:
a. decreverat (,er hatte erteilt'): arteilta (,er erteilte')
b. fluxerat (,es war geflossen'): flôz (,es floss')
(glJun 201)
(ibd. 205)

Einzelsprachlich entwickelten die jüngeren germanischen Sprachen neben anderen periphrastischen Formen das periphrastische Perfekt mit haben und sein. Natürlich ist das haben-Perfekt der Form nach der Prädikativkonstruktion haben mit einem PII als sekundärem Prädikat nachempfunden. Hierbei spielten jedoch verschiedene, teils voneinander unabhängige Reanalyse- und Reinterpretationsvorgänge eine Rolle. Zur Veranschaulichung folgt eine Synopse verschiedener Versionen von $L k$ $(19,20)$ :

$$
\begin{aligned}
& \text { a. domine ecce moneta tua quam habui }{ }^{7} \\
& \text { Herr sieh Geld deines } R E L-A C C-F \text { hab-PERF } \\
& {[\text { repositam in sudario }]^{8}} \\
& \text { aufbewahrt- } A C C-F \text { in Schweißtuch }
\end{aligned}
$$

5. Zugegebenermaßen ist das Perfekt im Lateinischen Erzähltempus der Vergangenheit. Diese Funktion erfüllt im Ahd. wie im Nhd. das Präteritum. Dennoch fällt auf, dass der eng an der Vorlage arbeitende Tatianübersetzer das Präteritum verwendet, zumal hier das Perfekt kontextuell nicht unpassend wäre.

6. Glossae Junii in Nyerups symbolae ad literaturam teuton. antiquiorem. Kopenhagen 1787. Nach Grimm (Dt. Gramm: 176).

7. Man beachte, dass das synthetische Perfekt von habere in der lateinischen Vorlage ein analytisches Perfekt mit habere als Auxiliar ausschließt.

8. Die Klammerung zeigt die Konstituenz des mit dem Bezugsnomen kongruierenden Partizips als freies Prädikativ an. Dies steht im Gegensatz zu unserer in 0 vorgeschlagenen 
b. frauja, sai, sa skatts peins panei habaida Herr sieh diesen Schatz deinen den hab-PRÄT [galagidana in fanin] gelegt- $A C C-N$ in Schweißtuch

c. herro, senu thin mna, thia ich habeta Herr sieh dein Geld $R E L-A C C-F$ ich hab-PRÄT [gihaltana in sueizduohhe] gehalten- $A C C-F$ in Schweißtuch ,Herr, sieh dein Geld, das ich bei mir hatte, aufbewahrt in einem Schweißtuch.'

d. Hier ist dein Pfund. Welchs ich habe im Schweistuch behalten

e. Ich habe es in ein Tuch eingebunden und aufbewahrt.

(Hausbibel)

Während im Gotischen und frühen Ahd., wie oft auch im Lateinischen, Prädikativkonstruktionen mit PII zur Wiedergabe von perfektischem Aspekt gebräuchlich waren, wird nach nhd. Sprachgebrauch hier die reguläre Perfektkonstruktion verwendet. Perfektisch interpretierbare Sätze mit haben und PII als nachgestelltem Attribut oder Prädikativ gibt es in den frühen germanischen Quellen in der Tat jedoch zahlreiche. So auch im ahd. Tatian, wo diese Sätze teils der lat. Vorlage entsprechen, teils aber auch das lat. Perfekt wiedergeben. Dass das folgende Beispiel den Satzbau der lateinischen Vorlage nicht exakt wiedergibt und dennoch dieselbe Prädikativkonstruktion verwendet, lässt darauf schließen, dass die Konstruktion haben + Prädikativum auch im Ahd. voll produktiv war (vgl. Morris 1988).

a. arborem fici habebat quidam plantatam in Baum Feige-GEN hatte jemand gepflanzten in vinea sua

Weingarten seinem

(Vulg: Lk 13,6)

b. phígboum habeta sum gipflanzotan in sinemo uuingarten

(Tat 102,2)

,Einen Feigenbaum hatte jemand, (der war) gepflanzt in seinem Weingarten.

Im folgenden Beispiel gibt dieselbe Prädikativkonstruktion, wie schon oben in (7b), das Perfekt eines lat. Deponens wieder:

Analyse der Perfektkonstruktion, wo das Partizip eine syntaktische Einheit (Verbalkomplex) mit der flektierten Verbform bildet. 

a. omnis qui viderit jeder REL sehen-FUT-3.Sg Weib zu begehren eam, iam moechatus est eam in corde suo. sie schon geschändet $A U X$ sie in Herzem seinem

(Vulg: Mt 5,27)

b. iogiuuelih thie thar gisihit uuîb sie zi gêronne iu jeglicher $R E L$ da ansieht Weib sie zu begehren schon habet sia forlegana in sinemo herzen hat sie geschändet- $A K K-S g$-fem in seinem Herzen

(Tat 28,1)

,Wer eine Frau ansieht, um sie zu begehren, hat sie schon geschändet (freies Prädikativ) ${ }^{9}$ in seinem Herzen.

$\mathrm{Zu}$ beachten ist, dass das PII forlegana prädikativ auf sia bezogen ist, das wiederum das Objekt von habet darstellt. In der nhd. Version in der Hausbibel ist eine solche Lesart ausgeschlossen.

(13) a. Wer ein Weib ansiehet, ihrer zu begehren, der hat schon mit ihr die Ehe gebrochen in seinem Herzen. (Luther)

b. Wer eine Frau auch nur lüstern ansieht, hat in seinem Herzen schon Ehebruch mit ihr begangen. (Hausbibel)

Unser Hauptargument dafür, dass in ahd. Sätzen wie diesem noch keine echte Perfektkonstruktion vorliegen kann, wird in diesem Beitrag sein, dass ein vollständiges Paradigma analytischer Perfektflexion im Ahd. erst ca. zwei Jahrhunderte später vorlag. Sätze wie der obige waren immer nur mit transitiven Verben möglich, deren PII prädikativ auf ein Referenznomen bezogen werden konnte. Erst im Schrifttum Notkers III des Deutschen (um 1000 n. Chr.) ist das Perfekt mit intransitiven Verben und Verben bezeugt, die ein Dativobjekt selegieren (s. u. 2.3). Des Weiteren indiziert u. E. die Nominalkongruenz am PII, dass dieses prädikativ verwendet ist, also nicht das Hauptverb des Satzes darstellt. Ausgangspunkt unserer Argumentation ist das so genannte habere-Perfekt im klassischen Latein.

9. Um das Prädikativ im Nhd. eindeutig zu machen, könnte man es hier alternativ durch als Geschändete wiedergeben, was dem Satz possessive Lesart verleiht. Jacob (z. B. 1998: 113) kritisiert in seinen Arbeiten zum habere-Perfekt im Lateinischen u. E. zurecht, dass die possessive Lesart derartiger Prädikativkonstruktionen nicht diejenige gewesen sein kann, die tatsächlich Grundlage der Grammatikalisierung war. Auch hier ist diese Interpretation hinsichtlich der perfektischen lat. Vorlage nicht wirklich befriedigend. Eine spezifische Modal- oder Aspekt-Periphrase auf nominaler Basis, wie von Jacob für das Lat. vorgeschlagen, lässt sich im Nhd. jedoch nicht leicht nachvollziehen - wenn man sie nicht mit dem paradigmatischen periphrastischen Perfekt übersetzt, für das es zur Zeit der Tatianübersetzung keine wirklich eindeutigen Belege gibt. 


\subsection{Habere + Partizip II im Lateinischen}

Im Gegensatz zu traditionellen Annahmen (vgl. Brinkmann 1931; Grandgent 1962) muss u. E. davon ausgegangen werden, dass ein habenPerfekt in der klassischen Periode des Lateinischen noch nicht existierte. Auch in der Vulgata wurden ausschließlich synthetische Perfektformen verwendet (abgesehen vom bereits grammatikalisierten Gebrauch der Kopula mit Deponentia und ähnlichen Konstruktionen). Ein periphrastisches Perfektparadigma mit habere entstand erst im Spätlatein, und zwar, ähnlich wie im Deutschen, durch Grammatikalisierung und Reanalyse von implizit perfektischen attributiven oder prädikativen Konstruktionen mit dem possessiven Vollverb. Derartige Konstellationen sind im klassischen Latein oft bezeugt.

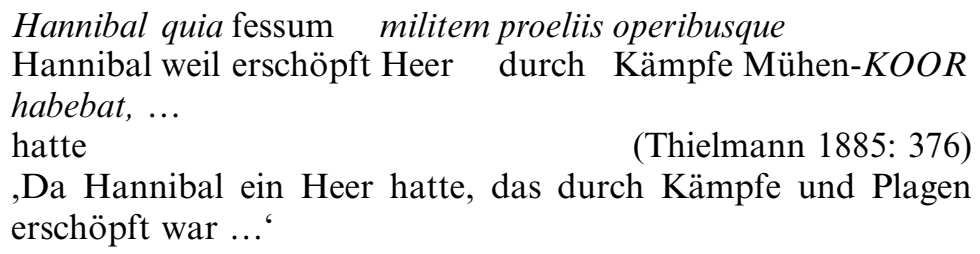

,Da Hannibal ein Heer hatte, das durch Kämpfe und Plagen erschöpft war ...'

Derartige Konstruktionen werden von vielen Autoren (z. B. Grandgent 1962: 54) als frühe Erscheinungsformen des periphrastischen Perfekts betrachtet. Bereits zu klassischer Zeit gibt es in der Tat Strukturen, die aus heutiger Sicht ambig sind (vgl. Grandgent 1962: 54):

$$
\begin{aligned}
& \text {... quid Athenis exquisitum habeam } \\
& \text { was Athenern ausgesucht(es) habe-1.KONJ }
\end{aligned}
$$

(Cato der Ältere, n. Thielmann 1885: 516) ,... was ich für die Athener an Ausgesuchtem habe.

Solche Sätze gemäß heutigem Sprachgebrauch als Perfektsätze lesen zu wollen, gehört u. E. jedoch zu den Missgriffen der lectio moderna. In der klassischen Periode lag zu keiner Zeit ein produktives Perfektparadigma mit habere als Auxiliar vor. Besonders wenn, wie im folgenden Satz, das perfektive PII in Kombination mit dem lat. synthetischen Perfekt verwendet wird, ist es evident, dass kein periphrastisches Perfekt vorliegen kann.

$$
\begin{aligned}
& \ldots \text { quam semper cognitam habui } \\
& \text { was- } A K K-F \text { immer gedacht- } A K K-F \text { hab- } P E R F
\end{aligned}
$$

(Quelle unbek.; n. Grandgent 1962: 55)

,... die ich immer als Gedachte(s) (in Gedanken?) gehabt habe. 
Dies zeigt zwar, dass im Lateinischen oftmals aspektuell interpretierbare Prädikativkonstruktionen verwendet wurden, wo in ähnlichen Kontexten moderne romanische Sprachen ein periphrastisches Perfekt verwenden würden. Der Annahme, dass hier der Vorbote einer periphrastischen Perfektkonstruktion vorläge, kann aus systematischer Sicht aber nur widersprochen werden.

Ein weiteres mögliches Argument gegen die Interpretation der Konstruktion habere + PII als Beispiel periphrastischer Flexion ist die Nominalkongruenz am PII, die es als Attribut oder Prädikativ ausweist. Nach Ramat (1982: 195) ist im Spätlateinischen das habere-Perfekt eben dadurch zu identifizieren, dass die Nominalkongruenz verloren gegangen ist.

$$
\begin{aligned}
& \text { a. litteram scriptam habeo } \\
& \text {,ich habe einen Brief im geschriebenen Zustand“ }
\end{aligned}
$$

(Ramat 1982: 195)

b. litteram scriptum habeo $>$ frz. J'ai écrit une lettre (vs. une lettre écrite) ${ }^{10}$

Das entscheidende Indiz für die Existenz eines periphrastischen Perfekts als Flexionstyp ist u. E., dass es mit sämtlichen Vollverben gebildet werden kann. Um eine Konstruktion als Flexionstyp zu identifizieren, sollte sie zumindest zu einem gewissen Grad oder mithilfe von Suppletivformen paradigmatisch sein.

Um mit intransitiven Verben wie schlafen oder Verben mit Dativobjekt wie helfen ein periphrastisches Perfekt zu bilden, müssen diese zunächst einmal in der Lage sein, ein Partizip Perfekt zu bilden, damit sie in einer Konstruktion mit Auxiliar als Hauptverb fungieren können. Das PII unergativer Verben oder solcher mit Dativobjekt wurde im klassischen Latein jedoch gar nicht gebildet, da es ja nur in der betreffenden Konstruktion vorkommt. Es mussten also erst verschiedene strukturelle und kategorielle Reanalysen stattfinden, bis sich aus der vorhandenen Prädikativkonstruktion habere + PII die Perfektperiphrase entwickelte und das Verb haben als Auxiliar rekategorisiert wurde. Dies gilt gleichermaßen

10. An dieser Stelle müssen wir jedoch berechtigte Einwände aus der romanistischen Linguistik einräumen, dass z. B. im mod. Frz. sehr wohl perfektisch zu interpretierende habenKonstruktionen mit Nominalkongruenz am PII existieren, wie z. B. Je l'ai écrite, la lettre. Dies entspräche in etwa einer Terminativkonstruktion wie Engl. I have the letter written, meanwhile. Auf diese Weise lassen sich jedoch nicht sämtliche dem Frz. eigenen Fälle von Nominalkongruenz im Perfekt wegerklären, wie z. B. beim sein-Perfekt. Für das Deutsche lässt sich u. E. die Behauptung dennoch aufrecht erhalten, dass nach der Grammatikalisierung der Perfektkonstruktion keine PII mit nominalen Merkmalen zu finden sind. 
für die romanischen wie für die germanischen Sprachen. In der vorliegenden Arbeit wollen wir die entscheidenden Argumente anhand des Ahd. ausführen.

2.3 Das Auftauchen des periphrastischen Perfekts im Althochdeutschen Im Folgenden wollen wir die Reanalysevorgänge, die letztlich die Rekategorisierung eines verbum possessivum als Auxiliar bewirkten, anhand des Ahd. modellieren, das u. E. erst ab dem 9. Jh. n. Chr. eine Entwicklung vollzog, die mit etwas früher zu datierenden Prozessen in den romanischen und anderen germanischen Sprachen konvergent war und nicht, wie noch von Grønvik (1986) vorgeschlagen, die Perfektkonstruktion aus dem Spätlatein entlehnte.

Im Deutschen gab es, soweit die Quellen dies zu erschließen erlauben, erst ab Notker haben + PII mit Verben, die kein Akkusativobjekt regieren.

a. tar habet si imo geantwurtet sinero frago
,Da hat sie ihm auf seine Frage geantwortet.

b. so habet er gelogen

,So hat er gelogen.'

c. habe ich keweinot so filo ,Ich habe so viel geweint.'
(Notk I: 284, 26)

(Notk I: 544,29)

(Notk II: 15,30)

Wie bereits einleitend erwähnt, kann das Ahd. innerhalb des Westgermanischen hierbei als Nachzügler gelten. Entsprechende Beispiele finden sich im altsächsischen Heliand schon um 840 n. Chr.:
a. habde iru tho giholpan helag barn godes hatte ihr da geholfen heilig Kind Gottes
b. habda at them uuiha [...] gilibd hatte-3.Sg bei dem Tempel gelebt

Der Heliand ist eine Evangeliendichtung, also keine Übersetzung, die ihre Existenz der Missionierung der Sachsen durch die karolingischen Franken verdankt (vgl. Öhl 2008). Da es sich um freie Dichtung handelt, muss also das Altsächsisch, in dem er verfasst wurde, in der vorliegenden Form bereits existiert haben, als begonnen wurde, lateinische Texte zu rezipieren. Dies allein sollte schon als Argument gegen die Annahme gelten, dass das haben-Perfekt entsprechenden Konstruktionen in mittellateinischen Texten nachgebildet worden wäre. ${ }^{11}$

11. Soweit bekannt, gab es keine lat. Vorlage für die Helianddichtung. Zudem ist es nicht evident, dass im Mittellatein des frühen 9. Jh. PII intransitiver Verben mit habere verwendet wurden. 
Der entscheidende Unterschied zu den in 2.2 angeführten lateinischen Beispielen mit habere + PII ist, dass letzteres, und nicht das Verb haben, den Selegierungsrahmen bestimmt und somit das Hauptverb darstellt. Selegiert aber haben die Objekte, kann es nur selbst das Hauptverb sein, und es liegt keine periphrastische Konstruktion vor - wie es auch in folgendem nhd. Satz der Fall ist:

(20) Wir haben noch vieles, das erledigt werden muss.

In den folgenden Abschnitten entwerfen wir zunächst ein Erklärungsmodell für Grammatikalisierungsprozesse, das u. E. geeignet ist, die Auxiliarisierung von haben zu erfassen. Im Anschluss daran ermitteln wir die syntaktischen und semantischen Bedingungen, die in den jeweils relevanten zeitlichen Abschnitten die Grammatikalisierung von haben als funktionelles Element ermöglichten. Abschließend werden wir versuchen, den Wandelprozess als Ganzes zu modellieren.

\section{Grammatikwandel und kognitive Ökonomie}

\subsection{Sprachwandel und Parametrisierung}

Unter Grammatikalisierung versteht man bekanntlich einen Sprachwandelprozess, in dessen Verlauf eine autonome lexikalische Einheit allmählich die Funktion einer abhängigen grammatischen Kategorie erhält. Der Terminus wurde von Meillet (1912) geprägt (vgl. Lehmann 1995: 1). Das Phänomen wurde in der moderneren Sprachwissenschaft zunächst aus funktionalistischer Sicht beschrieben (z. B. Meillet 1912, Kuryłowicz 1965, Hopper \& Traugott 1993, Lehmann 1995). Formalistische Erklärungen von Sprachwandelprozessen aus der Sicht der generativen Grammatik (z. B. Lightfoot 1979, 1991) führten auch zu verschiedenen formalen Erklärungsansätzen für Grammatikalisierungsphänomene (z. B. Abraham 1992b, 2000, 2004; Roberts 1993; Roberts \& Roussou 2003; Gelderen 2004).

Im Mittelpunkt der formalen Ansätze steht traditionell die diachrone Reanalyse der (morpho-)syntaktischen Struktur, mit der oftmals die kategoriale Reanalyse (,Rekategorisierung') lexikalischer Einheiten zu funktionalen Elementen einhergeht. Die Reinterpretation eines Vollverbs wie haben zum Auxiliar, zusammen mit der Reanalyse einer Prädikativkonstruktion, ist hierfür ein einschlägiges Beispiel.

In der nativistisch konzipierten generativen Syntaxtheorie gilt hierbei die Grundannahme, dass diese Reanalyse beim Generationenwechsel durch abweichende Parametrisierung sprachlicher Prinzipien während des Grammatikerwerbs durch die Folgegeneration zustande kommt. Diese Art der generativen Grammatik ist eine Theorie des kognitiven 
Sprachmoduls und seiner Rolle für die menschliche Sprachkompetenz und den Erwerb einer (oder mehrerer) Erstsprachgrammatik(en) (firstlanguage-acquisition; vgl. Chomsky \& Lasnik 1993: 506 f.; fortan $\mathrm{L}_{1} \mathrm{~A}$ ). Die Funktionsweise dieses Moduls ist durch inhärente Prinzipien determiniert (UG, Universalgrammatik). Sprachprinzipien und deren Parameter bilden die Grundlage der einzelsprachlichen Grammatik, indem auf diese während $\mathrm{L}_{1} \mathrm{~A}$ durch UG (der Genotyp aller menschlichen Sprachen, hier als kognitives Modul aufgefasst) der Verlauf sprachlicher Erfahrungen abgebildet wird (Parametrisierung; vgl. Öhl 2006: 231). Als einfaches Beispiel für Parametrisierung mag die Wortstellung VO vs. OV dienen, die durch den Direktionalitätsparameter ${ }^{12}$ in der VP erklärbar ist. U. a. legt das Kind beim Spracherwerb fest, ob in der Grammatik seiner Sprache die Objekte dem Verb vorangehen oder aber folgen werden.

a. Paul has [vp eaten [DP an apple]]

b. Paul hat [vP [DP einen Apfel] gegessen]

Parametrisierung aufgrund sprachlichen Inputs kann aber konkret nur dann stattfinden, wenn dieser robust ist: Nach Lightfoot (1991: 19) ist Triggering eine Funktion aus Salienz und Frequenz sprachlicher Muster, die den Parameterwert zu erschließen erlauben. Beim Spracherwerb wird die Grammatik G2 durch die Zuweisung konvergenter Strukturen an das linear wahrgenommene sprachliche Produkt der Grammatik G1 rekonstruiert (vgl. Öhl 2006: 231). Soll G2 mit G1 identisch sein, muss ausgeschlossen werden, dass abweichende Daten zum Trigger werden. Abweichende Daten sind jedoch in der gesprochenen Sprache, die dem Kind als Input dient, nur gewöhnlich. Zudem, und hierauf beruht eine der grundlegenden Annahmen unseres Sprachwandelmodells, bewirkt die Manipulation des sprachlichen Outputs durch teils konventionalisierte Regeln der Performanz oftmals die abweichende Rekonstruktion der Parameterwerte der Elterngrammatik, was dann zur Reanalyse der syntaktischen Struktur führt (vgl. Öhl \& Korn 2006: 174 ff.).

\subsection{Reanalyse und die Gradualität von Grammatikalisierungsprozessen}

Da neue Strukturbildungstypen infolge diachroner Reanalyse in der Regel spontan auftauchen und eine alte Regel in der Grammatik der Folge-

12. Wir wollen an dieser Stelle darauf hinweisen, dass wir trotz Kaynes (1994) Antisymmetriehypothese den Direktionalitätsparameter nicht als widerlegt ansehen. Entsprechende Argumente sind in Öhl (2003: 104 ff.) aufgeführt. Auch Radford et al. (2006: 350) verwendet in seiner Einführung Direktionalität als Beispiel für die Parametrisierung. Für die Parametrisierung der Direktionalität auch im minimalistischen Rahmen argumentiert u. a. Fukui (2002: 399 ff.). 
generation oft schnell verdrängt wird, zählen Reanalysen zu den so genannten abrupten Sprachwandeln. Funktionalistische Forscher sehen hier einen Gegensatz zur graduellen Natur des Grammatikalisierungsprozesses (s. v. a. Haspelmath 1994, 1998), bei dem durch schrittweises semantisches Verblassen die grammatische Funktion eines Ausdrucks in den Mittelpunkt tritt, bis schließlich (sofern der Ausdruck vollständig grammatikalisiert wird) daraus ein Grammem entsteht. Des Weiteren wird kritisiert, dass Grammatikalisierung mehr sein müsse als formale Reanalyse, da die Sprecher durch sie die Funktionsfähigkeit ihres Sprachsystems verbessern. Diese Vorstellung basiert auf funktionalistischen Konzepten des Sprachwandels wie dem von Coseriu (1974, 1975; vgl. auch Erben 1976). An dieser Stelle kann nicht die gesamte methodologische Diskussion der Erklärungsadäquatheit des jeweiligen Modells referiert werden. Zum Verständnis unserer Vorgehensweise wollen wir jedoch einige klärende Anmerkungen voranschicken.

Die Notwendigkeit der strengen Trennung von Grammatikalisierung und Reanalyse, graduellem und abruptem Wandel sowie funktionaler und formaler Reinterpretation von Ausdrücken, wie sie vor allem von Haspelmath (1998) gefordert wird, sehen wir nicht. Wir schließen uns Abraham (1992b, 2000, 2004) an, indem wir annehmen, dass Reanalyse ein durchaus relevanter Mechanismus in Grammatikalisierungsprozessen sein kann.

Zwar ist unser Grammatikalisierungsbegriff nicht rein formal, wie beispielsweise derjenige von Roberts (1993) und Roberts \& Roussou (2003), die davon ausgehen, dass rein strukturelle Reanalysevorgänge auf arbiträre Art in Grammatikalisierung münden können. Für ein Modell mit adäquatem Erklärungspotential recht viel versprechend erscheinen uns dagegen die Vorschläge von Gelderen (1993, 1996, 2004). Ihr Ansatz ist an funktionalistische Erklärungen angelehnt, denn sie betrachtet wie diese Grammatikalisierung nicht als spontanen, rein formalen Kategoriewechsel, sondern als schrittweisen Prozess, bei dem lexikalische Bedeutung verloren geht und grammatische Funktion übernommen wird (nach Gelderen 1993: 169). In ihrem Modell wird der Übergang von einer Kategorie in die andere vollzogen, wenn der Grammatikalisierungsprozess mit der strukturellen und kategoriellen Reanalyse abgeschlossen wird (vgl. Gelderen 1996, 2004). Sie demonstriert dies am Beispiel des Infinitivmarkers to, der im Altenglischen Präposition war, im Mittelenglischen auxiliarähnliche Funktion annahm und ab dem frühen Neuenglischen zusammen mit den auxiliarisierten Modalen (z. B. will, shall, should) der Lexikalisierung der funktionalen Kategorie T(empus) diente (Gelderen 1996: 40 f.). Einen solchen Ansatz kann man integrativ nennen, da er funktionale und formale Ansätze der Erklärung von Sprachwandel in sich vereint: Funktionell bedingte Variation der Perfor- 
manz im Randbereich der Grammatik schafft die Voraussetzung für parametrischen Wandel der Kerngrammatik.

Wir schließen uns der nativistischen Annahme an, dass die grundlegenden Regeln der einzelsprachlichen Grammatik nur beim Generationenwechsel verändert werden oder verloren gehen können (abduktiver Wandel; vgl. Andersen 1973: 774 ff.). ${ }^{13}$ Dies beinhaltet die (unbewusste) Reanalyse syntaktischer Strukturen der Elterngrammatik durch die spracherwerbende Generation, wodurch neue syntaktische Regeln entstehen, die erst in der so neu entstandenen Grammatik Anwendung finden. Die Möglichkeit derartiger Neubildung von Regeln auf der Ebene der Performanz ist einem Sprecher nicht gegeben. Ein Weg, der Sprechern jedoch zur Grammatikveränderung offen steht, ist die Regeladdition (cf. Kiparsky 1975: 241 ff.), ${ }^{14}$ sofern diese nicht den bereits vorhandenen Regeln des Sprachsystems zuwiderläuft. Sie findet auf der Ebene der Sprachnorm (im Sinne Coserius 1975) statt, betrifft also nicht die Kernbereiche der internalisierten generativen Grammatik, und dient in der Regel der Verbesserung des ,Funktionierens' der Sprache, also in der Regel entweder der Erleichterung der Performanz oder der Erhöhung der Expressivität.

Der Spielraum für derartige Regelmanipulationen durch den Sprecher gehört zur so genannten konditionalen Frage der diachronen Linguistik, der Frage nach den Bedingungen, die für einen bestimmten Wandel vorhanden sein müssen (cf. Coseriu 1975). Diese betrifft die Restriktionen durch das vorhandene Sprachsystem, sowie das Inventar von sprachlichen Ausdrücken, die für performanzbasierten bzw. parametrischen Wandel zur Verfügung stehen. So zeigen die ahd. Quellen bezüglich der Grammatikalisierung des Perfektauxiliars klar, dass aspektuell interpretierbare Konstruktionen mit haben + PII zunächst auf Kontexte beschränkt waren, in denen mit dem PII über ein direktes Objekt von haben sekundär prädiziert wurde (s. o. 2.3). Nachdem diese Art der Aspektmarkierung jedoch zu einem hohen Grade konventionalisiert war, wurde haben als Auxiliar rekategorisiert, und es konnten Perfektkonstruktionen mit intransitiven Verben gebildet werden. Dies stellt eine Art der Regelmanipulation dar, die im Rahmen der Performanz schlichtweg unmöglich ist.

Nicht jede Veränderung in der Sprache basiert zwangsläufig auf einer Veränderung der Kerngrammatik. Jedoch schafft so genannter Norm-

13. Das Modell abduktiven Wandels wurde von Andersen (1973) zunächst für phonologische Wandelphänomene entwickelt, lässt sich jedoch auch auf morphosyntaktischen und lexikalischen Wandel ausweiten; vgl. z. B. McMahon (1993: 122, 177). Es beschreibt grob gesagt die Entstehung neuer Strukturen durch beim Spracherwerb abweichend generalisierte Regeln.

14. Auch diese Schlussfolgerung wurde zunächst anhand phonologischen Wandels gezogen. 
wandel (z. B. Konventionalisierung) entscheidende Bedingungen, unter denen ein Systemwandel ausgelöst werden kann. Lightfoot (1991: 160) weist explizit darauf hin, dass die Konventionalisierung von Ausdrucksfunktionen, die in funktionalistischen Modellen seit jeher als die maßgebliche Quelle sprachlichen Wandels gilt, in der generativen Grammatik wiederum zu gering eingeschätzt wird. Die zunehmende Häufigkeit von Ausdrücken in veränderter Funktion erweckt den Anschein graduellen Wandels der Grammatik, tatsächlich wird innerhalb von ein- und derselben Grammatik nur der Schwerpunkt des Gebrauchs von Ausdrucksfunktionen verlagert. Es handelt sich also um graduellen Wandel von Konventionen der Performanz. Lightfoot (1991) demonstriert dies anhand des Verbstellungswandels von OV zu VO im Englischen (vgl. unser obiges Bsp. 21, sowie Öhl 2006: 236 ff.)

Der Wandel der Kerngrammatik findet also abrupt und während des Spracherwerbs statt, dann nämlich, wenn die der spracherwerbenden Generation verfügbaren Daten bewirken, dass ein Parameter anders gesetzt wird. Dieser Wandel ist als strukturelle oder kategoriale Reanalyse beschreibbar. Im Verlauf eines Grammatikalisierungsprozesses kann eine solche wiederholt stattfinden, ${ }^{15}$ wobei typischerweise jedes Mal lexikalischer Gehalt von Ausdrücken verloren geht, oft einhergehend mit der substantiellen Reduktion.

Dies bietet eine Erklärung für wesentliche Eigenschaften des so genannten Grammatikalisierungskanals (engl. cline), nämlich das graduelle semantische Verblassen und die Unidirektionalität. Die Inhalte vorangehender sprachlicher Daten sind nämlich für den Grammatikerwerb späterer Generationen schlichtweg nicht mehr zugänglich und können so keine Erstellung von Regeln mehr bewirken. Einwände, wie der von Haspelmath (1994: 319), dass die Erklärung der Grammatikalisierung durch Reanalyse die Umkehr des Prozesses erlauben sollte, scheinen also nicht gerechtfertigt. Sprachwandel in der generativen Grammatik ist nicht rein arbiträr; Voraussetzung ist, dass ein entsprechender Auslöser in den Sprachdaten des Inputs für den Spracherwerb vorhanden sein muss. Dieser ist aber bei derartigen Reanalysen nicht gegeben, und es wäre überprüfenswert, ob bei den wenigen Fällen, die, degrammatikalisierungsverdächtig' sind (vgl. Lehmann 1995: 16 ff.), entsprechende Reanalysen durch den für die Sprachlerner zugänglichen Input begünstigt sind.

15. Interessanterweise spricht auch Haspelmath (1998: 345 f.) von einer Folge von ,Mikroreanalysen' im Zusammenhang mit dem Erwerb stärker grammatikalisierter Formen durch eine Folgegeneration von Sprechern. Allerdings gibt er diesen weitaus weniger Gewicht als den performanzbasierten Wandeln, die seiner Ansicht nach den Kern des Grammatikalisierungsprozesses ausmachen. 
Die deskriptiv feststellbare Gradualität, die in funktionalen Arbeiten zum Syntaxwandel (cf. Chung 1977, Langacker 1977) die Annahme einer Kette von Regelveränderungen (Langacker 1977: 58) begründete, was schließlich zum Konzept der natürlichen Serialisierung von Reanalysen führte (Lehmann 1973; Vennemann 1974), lässt sich auf diese Weise mit den Mitteln formal/nativistischer und performativ/funktionaler Konzepte in Kombination als konditional im Sinne Coserius (1975) erklären. Reanalyse kann dann stattfinden, wenn der sprachlichene Input eine Reinterpretation von Strukturen oder Kategorien zulässt. Performative Manipulation von Strukturen, Bedeutungsmerkmalen oder der Anwendung von Ausdrücken kann stattfinden, wenn das bestehende grammatische System dies erlaubt. Vorangehende Regelmanipulation schafft jedoch häufig erst die Bedingung für die diachrone Reanalyse. Somit kann es zu einer alternierenden Abfolge von performanzbasierten und durch den Spracherwerb zu erklärenden Wandeln kommen.

\subsection{Sprachwandel und kognitive Ökonomie}

Wir gehen zudem davon aus, dass das Spannungsverhältnis zwischen funktionalen und formalen Aspekten morphosyntaktischen Wandels vor dem Hintergrund eines differenzierten Modells kognitiver Ökonomie beleuchtet werden muss. Hierbei sind verschiedene Formen der Ökonomie relevant, deren Konkurrenz kennzeichnend für Grammatikalisierungsprozesse ist. Es handelt sich um das in der historischen Sprachwissenschaft traditionell angenommene Wechselspiel von Ökonomie und Expressivität (vgl. hierzu auch Hopper \& Traugott 1993: 65f.), das von Martinet (1981) wie folgt formuliert wurde: „Die gesamte Sprachentwicklung wird bestimmt von dem stets vorhandenen Widerspruch zwischen den kommunikativen und den expressiven Bedürfnissen des Menschen einerseits und andererseits seiner Neigung, seine geistige und physische Aktivität auf ein Minimum zu beschränken." (Martinet 1981: 85).

Diese Feststellung wurde von Martinet ursprünglich für performanzbasierten Wandel getroffen. Von Seiten der generativen Grammatik wurde darüber hinaus argumentiert (v. a. Roberts 1993, Roberts \& Roussou 2003), dass auch Kinder beim Spracherwerb insofern kognitiv ökonomisch vorgehen, als sie dem sprachlichen Input eine möglichst wenig aufwändige Struktur zuweisen. Dies wurde bereits von Roberts (1993) als universelle Spracherwerbsstrategie formuliert:

\section{Least Effort Strategy (LES)}

Representations assigned to sentences of the input to acquisition should be such that they contain the set of the shortest possible chains (consistent with (a) principles of grammar, (b) other aspects of the trigger experience).

(Roberts 1993: 10) 
Dieses Vorgehen führt oftmals zu Grammatikwandel, wenn nämlich zwei mit dem Input konvergente Strukturen möglich sind, von denen die ursprüngliche die unökonomischere ist. Hierdurch lässt sich beispielsweise die Reinterpretation des Vollverbs haben zu einem Flexionselement erklären, indem zu einem bestimmten Zeitraum beim Spracherwerb Sätzen eine Struktur zugewiesen wurde, in der das aspektindizierende Element im Kopf der Tempusphrase generiert wurde, weil diese Struktur einfacher zu erzeugen war als eine, in der das Element durch Permutation in die entsprechende funktionale Position geriet.

Was aber sind die von Roberts erwähnten , anderen Aspekte' der Parameterfestlegung? Wir möchten in diesem Beitrag unter anderem dafür argumentieren, dass Sprachlerner komplexe sprachliche Ausdrücke systematisch auf die Spezifikation von Bedeutungsprimitiven hin zu strukturieren versuchen. Hierbei kann es zur Lexikalisierung funktionaler Kategorien kommen, die eine Struktur ursprünglich nur implizierte. Hierzu kann wiederum das haben-Perfekt als Beispiel dienen: Die in den jeweiligen Sätzen durch das PII vorhandene Bedeutungskategorie des perfektiven Aspekts in Kombination mit der impliziten Vorzeitigkeit kann u. E. dazu geführt haben, das Verb haben als Element zur Lexikalisierung der funktionalen Kategorie T zu rekrutieren, was schließlich die Rekategorisierung als Auxiliar bedeutete.

$\mathrm{Da}$ die Operation mit uninterpretierbaren Ausdrücken nicht kognitiv ökonomisch sein kann, sollte optimierte Expressivität ebenso ein kognitives Ökonomieprinzip sein, wie das des geringen generativen Aufwands, das sich in der Erzeugung möglichst wenig aufwändiger Strukturen niederschlägt. Obgleich die Erzeugung expliziter komplexer Ausdrücke kognitiv aufwändig ist, sind minimale Strukturen oftmals nicht explizit genug, um ökonomisch verarbeitet zu werden. Es müssen also zwei maßgebliche, aber konkurrierende Prinzipien kognitiver Ökonomie existieren, die zwangsläufig auch den Spracherwerb bestimmen:

\section{Prinzipien kognitiver Ökonomie}

a. Strukturen sind hinsichtlich des generativen Aufwands minimal.

b. Strukturen sind hinsichtlich der logischen Interpretation hinreichend spezifiziert.

Als Konsequenz von (23a) kann eine tendentielle Redundanzbeschränkung angesehen werden, die die morphologische Realisierung funktionaler Kategorien betrifft und z. B. dafür verantwortlich ist, dass $\varnothing$-Morpheme existieren können, ${ }^{16}$ bzw. nicht lokal realisierte funktionale Köpfe in der syntaktischen Struktur:

16. Diese Annahme diskutieren wir hier nicht im Detail, verweisen jedoch auf bekannte Ökonomiebedingungen der Wortbildung, wie die Synonymiebeschränkung oder andere Redundanzregeln. 
Es ist aus unserer Sicht nahe liegend, dass auch die Performanz vom Zusammenspiel dieser Prinzipien bestimmt ist, da sie zum einen die wenig aufwändige Sprachproduktion bevorzugt, zum andern aber die erfolgreiche Kommunikation zum Ziel hat (vgl. auch Haspelmath 1998: 320 f.). Wenn, wie wir annehmen, derartige Ökonomieeigenschaften der menschlichen Sprache auf angeborenen Prinzipien des menschlichen Kognitionssystems beruhen, müssen Sprachlerner zwangsläufig (unbewusst) voraussetzen, dass sprachliche Strukturen diesen gehorchend erzeugt werden. Also wenden sie diese Prinzipien (unbewusst) an, wenn sie die Elterngrammatik rekonstruieren.

Vor diesem Hintergrund lässt sich folgendes Szenario entwerfen: Wenn das spracherwerbende Kind sprachliche Strukturen interpretiert, sucht es zunächst nach logischen Primitiven, die potentiell durch die mehr oder weniger komplexen Ausdrücke im Satz enkodiert werden. Um die vorliegende Struktur zu entschlüsseln, entwirft es möglichst ökonomische Strukturen, die den ermittelten Bedeutungskomplexen zugrunde liegen können. Hierbei erlaubt das Kind bis zu einem gewissen Grad Unterspezifizierung, vorausgesetzt, dass die logische Interpretation gewährleistet ist. Konnte das Kind den sprachlichen Input erfolgreich entschlüsseln, legt es entsprechende Parameter seiner Grammatik fest, sofern es nicht massiv widersprüchlichen Daten ausgesetzt ist. Hierbei bedeutet ,erfolgreiche Entschlüsselung' jedoch nicht zwangsläufig, dass die elternsprachliche Grammatik exakt rekonstruiert wurde, sondern lediglich, dass den vorgefundenen Daten eine konvergente Struktur zugewiesen werden konnte. Weicht diese von den Bildungsregeln der Elterngrammatik ab, hat dies Grammatikwandel zur Folge. Veränderungen von Performanzkonventionen im Vorfeld, die sich aufgrund derselben Ökonomieprinzipien vollziehen, können eine solche Entwicklung nur begünstigen.

Somit liegt beim morphosyntaktischen Wandel eine grundsätzliche Dichotomie zweier Aspekte kognitiver Ökonomie vor, die für die Erzeugung sprachlicher Strukturen überhaupt bestimmend ist und zudem die Struktur des Lexikons beschränkt. Diese Konkurrenz kann folgendermaßen formuliert werden:

\section{Maxime kognitiver Ökonomie}

Generiere minimale Strukturen, die mit einer hinreichenden Spezifizierung logischer Interpretation konvergieren. ${ }^{17}$

17. Die Formulierung ist bewusst allgemein gehalten und vermeidet die Bezugnahme auf konkrete strukturelle Gegebenheiten, wie Gelderens (2004) SPEC $>$ Head- oder LateMerge-Bedingungen, da diese u. E. nicht alle strukturvereinfachenden Reanalysen fassen, die zur Entstehung der Perfektkonstruktion führten (vgl. 0). 
Sprachwandel folgen stets mehr dem einen oder dem anderen der beiden konkurrierenden Grundsätze, wodurch Kontraste zwischen extensiveren, aber expliziteren, und ökonomischeren, aber morphosyntaktisch weniger differenzierten grammatischen Systemen entstehen.

\section{Die syntaktischen Bedingungen für die Auxiliarisierung}

\subsection{Objektsprädikative und Verbalkomplexe}

Wenden wir uns nun wieder der diachronen Reinterpretation des Vollverbs haben zum Auxiliar zu, die mit der strukturellen Reanalyse von Sätzen mit freien Prädikativen einherging, wie sie auch im heutigen Deutsch noch verwendet werden.
a. Ob sie [vP die Getränke (auch) ${ }^{18}$ [AP PRO gekühlt ] (da) ha- $\left.\mathrm{ben}_{\mathrm{V}}\right]$ ?
b. Ob sie (auch) [vP die Getränke gekühlt haben $\left._{\mathrm{AUX}}\right]$ ?

Aus Sätzen der Struktur in (26a) entwickelte sich in althochdeutscher Zeit das heute gebräuchliche periphrastische Perfekt. Es ist ersichtlich, dass die Bildungsregeln des ursprünglichen Satzes nicht verschwunden sind, die Wandelprozesse jedoch zu einer neuen grammatischen Form geführt haben, die parallel zur ersten existiert (und heute wesentlich produktiver ist als diese).

Begünstigt wurde die Entstehung der Konstruktion haben + PII durch die morphologische Reduktion bei der Nominalkongruenz, die vermutlich phonotaktisch bedingt war. Der Bezug von Adjektiven und Partizipien zum Substantiv war ursprünglich sowohl in attributivem wie prädikativem Gebrauch durch Nominalkongruenz markiert. Dies war der Fall im Griechischen, Lateinischen und Gotischen, war vermutlich ein indogermanisches Phänomen, und auch im Vorläufer des Ahd. einmal so. $\mathrm{Zu}$ Beginn der ahd. Schreibperiode schwand die Nominalkongruenz und wurde sowohl attributiv wie prädikativ oft weggelassen, war also irregulär (vgl. Braune \& Eggers 1987: §247).
a. ther blintêr ward geboranêr
b. pist also gialtet man
(Otfr); Prädikativum mit NomK (Hild 41); Attribut ohne NomK

18. Da das Fokuspotential von Modalpartikeln der Identifizierung von Konstituenten dienen kann, zeigt im zweiten Satz der weite Skopus der Fokuspartikel auch die Verschiebung der syntaktischen Kategoriengrenze an. 
c. tot ist Hiltibrant, Heribrantes suno

(Hild 44); Prädikativum ohne NomK

Erst im Spät-Ahd. wurde die Nominalkongruenz beim Adjektiv im attributiven Gebrauch regelhaft, ebenso wie die Endungslosigkeit beim Prädikativum. Schon vorher müssen aber endungslose Prädikative oder nachgestellte endungslose Attribute als nominaler Teil eines komplexen Prädikats reinterpretiert worden sein, wobei das Hauptverb an eigener Semantik einbüßte. Dies geschah nicht nur in Konstruktionen mit dem Verb haben:

a. hialt uns [...] dar giborgan (,verborgen halten')

hielt uns da geborgen

,Er hielt uns dort verborgen.

(Otfr IV 55, 42)

b. in buah si iz duent [AP PRO zisamene gihaltan] zi habanne in Buch sie es tun zusammen gehalten zu haben ,Sie binden es zu einem Buch, damit es zusammen gehalten wird.'

(Otfr III 7, 54)

Hierzu muss sich aber ebenfalls eine strukturelle Reanalyse vollzogen haben. Der Vorgang kann als diachrone Bildung eines Verbalkomplexes (fortan VK) erklärt werden, bei dem das ursprüngliche Vollverb, das fortan den flektierten Kopf darstellte, semantisch abgeschwächt wurde. Es besteht also ein enger Zusammenhang mit den Funktionsverbgefügen im modernen Deutsch, die Polenz (1987: 172) sogar explizit mit dem haben-Perfekt vergleicht. Die gängigen Argumente für die syntaktische Kohärenz von Funktionsverb und dem prädikativen Nominalglied im Nhd. sind grundsätzlich auch für komplexe Prädikate wie oben gültig, so wie heute noch für idiomatische Gefüge mit haben. Dies zeigen, unter anderem, folgende Koordinationstests:

a. *Er hielt es verborgen und [ihm eine Rede].<smiles>CCCC</smiles>

kohärent

b. *Er hält den Kopf rasiert und [einen lustigen Hut in der

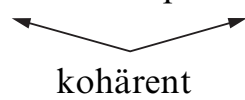

Hand].

a. *Er hat Recht und [ein loses Mundwerk].

kohärent 
b. *Er hat sie lieb und darum auch [zur Frau].<smiles>CCCC</smiles>
kohärent

c. *Er hat den Kopf rasiert und [einen lustigen Hut].<smiles>CCCC</smiles>

kohärent

Die Ungrammatikalität dieser Sätze rührt daher, dass nicht zwei koordinierte Konstituenten unter einem Vollverb haben vorliegen, sondern ein Verbalkomplex mit haben, der die zweite Konstituente nicht beinhalten kann. Man vergleiche:

(31) a. dass er es immer [vK verborgen halten] wird

b. dass er den Kopf immer [vK rasiert halten] will

a. dass er immer [vK (*ein loses Mundwerk und) Recht haben] wird

b. dass er sie immer [vK (*zur Frau und) lieb haben] wird

c. dass er seinen Kopf immer [vK rasiert gehabt] hat.

Im Gegensatz zur so genannten Small-Clause-Analyse der Reinterpretation sekundärer Prädikation zur Perfektkonstruktion, die wir im folgenden Absatz kurz vorstellen, gehen wir davon aus, dass sich die Auxiliarisierung von haben über den Zwischenschritt eines funktionalisierten Verbs in solchen Verbalkomplexen vollzog.

\subsection{SC-Analyse vs. VK-Analyse}

In Ansätzen wie Abraham (1986; 1991; 1992a) werden Sätze wie (30c) und (32c) (auch Stativsätze genannt; cf. Latzel 1977: 298) als SmallClause-Konstruktionen (SCs) analysiert. Abrahams Analyse der Grammatikalisierung basiert auf der Ansicht, der SC mit einem Kopf A sei als VP reanalysiert und dadurch V (haben) in einem einzigen Reanalyseschritt als Auxiliar reinterpretiert worden (Abraham 1991: 129, 1992a: 5). Dies entspräche in etwa folgendem Strukturwandel.

Wir vertreten stattdessen die Ansicht, dass bereits vor der Rekategorisierung von haben als Perfektauxiliar eine Reanalyse von PII und haben zum komplexen Prädikat stattgefunden haben muss.

Es gibt durchaus weitere Argumente gegen die Annahme, dass in Sätzen wie (32c) ein SC vorliegt. Beispielsweise stellt ein SC immer eine 

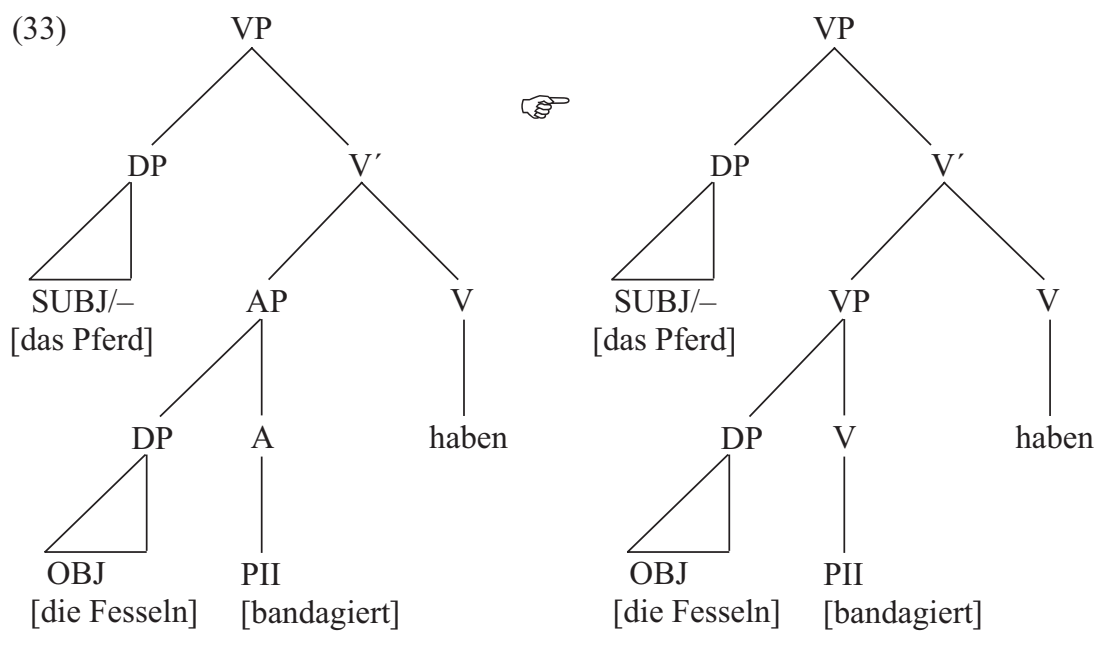

eigene Skopusdomäne für Satzadverbiale dar (vgl. Steube 1994: 249 ff.). Dies ist hier aber nicht der Fall:

a. Aus Unkenntnis wähnte sie [sc ihren Mann geschäftehalber in Berlin].

b. Seit damals hat ${ }_{\mathrm{i}} / \mathrm{hält}_{\mathrm{i}}$ er den Kopf aus Hygienegründen [vK rasiert $\mathrm{t}_{\mathrm{i}}$.

(VK)

Während in (34a) das Adverb geschäftehalber nur Skopus über das SC Prädikat in Berlin hat, hat das Adverbial aus Hygienegründen in (34b) Skopus über den Komplex rasiert haben/halten. Da der SC eine Konstituente bildet, kann man ihn (marginal) topikalisieren. Dies ist nicht möglich mit VK + Adverbial, da letzteres außerhalb des VK steht.

a. ?[sC Ihren Mann geschäftehalber in Berlin] wähnte sie (nur) aus Unkenntnis.

b. *Aus Hygienegründen [vK rasiert $\left.t_{i}\right]_{\text {hat }} / h /{ }^{2} l t_{i}$ er seit damals den Kopf.

Auf der anderen Seite kann man marginal den Komplex rasiert haben topikalisieren, während in Berlin und wähnen eindeutig niemals einen solchen Komplex bilden.

a. *In Berlin gewähnt hat sie aus Unkenntnis ihren Mann geschäftehalber.

b. ?[vK Rasiert haben/halten] könnte man den Kopf (z. B.) aus Hygienegründen. 
Interessanterweise werden Sätze wie jene oben in (30) wesentlich besser, wenn man statt einem Nominal eine weitere Phrase mit einem prädikativen Kopf koordiniert.

a. Er hat sie lieb und darum auch zur Frau genommen.

b. Er hat den Kopf rasiert und einen lustigen Hut auf.

Besonders (37a) zeigt, dass es sich nicht um zwei koordinierte SCs handelt, da beim koordinierten Teil eigentlich das Perfektauxiliar haben fehlt. Auch im zweiten Teil des Satzes (37b) ist fraglich, ob einen lustigen Hut auf eine Konstituente darstellt. Es handelt sich hier offensichtlich um Ellipse eines identischen Teils koordinierter Sätze (nämlich Gapping).
a. Er hat sie lieb und (er hat sie) darum auch zur Frau ge- nommen.
b. Er hat den Kopf rasiert und (er hat) einen lustigen Hut auf.

Als Charakteristika von Gapping gilt im Allgemeinen (Hartmann 2001: 158):

(39) Gapping ist ein PF-Phänomen, bei dem zusammen mit dem finiten Verb weitere Konstituenten des zweiten Konjunkts einer Koordinationsstruktur getilgt werden können, sofern sie mit Konstituenten im ersten Konjunkt identisch sind.

Gapping von haben wäre also auch bei unterschiedlichen Subjekten möglich:

(40) a. Er hat sie lieb und sie (hat) ihn darum auch zum Mann genommen.

b. Er hat den Kopf rasiert und sie (hat) einen lustigen Hut auf.

Was jedoch immer ausgeschlossen ist, ist Gapping des Vollverbs haben, wenn das desemantisierte haben vorausgeht (vgl. 32). Dagegen verhält sich haben in Stativsätzen, Partikelverbphrasen und der Periphrase hinsichtlich Gapping gleich. Dies spricht natürlich für deren Strukturgleichheit. Deshalb müssen wir (wie Bierwisch 1990; Haider 1994; vgl. auch Müller 2002 im HPSG-Rahmen) annehmen, dass in all diesen Konstruktionen haben und das nominale Element einen Komplex der Kategorie $\mathrm{V}$ bilden, in welchem eine nominale XP an den Kopf $\mathrm{V}$ adjungiert ist. 
(41)

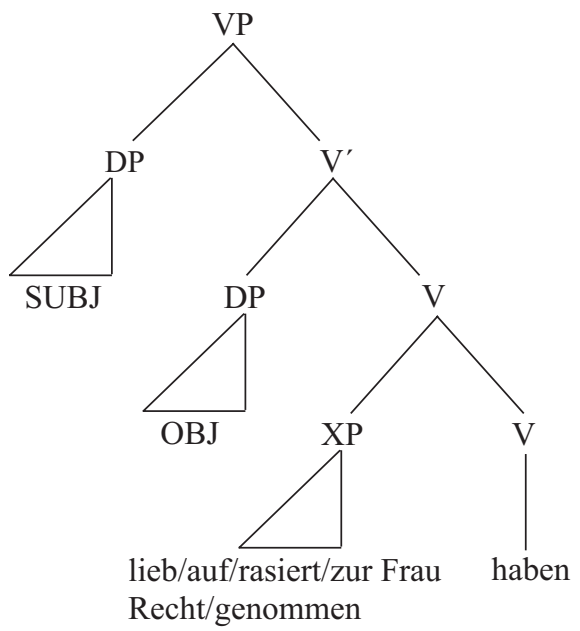

Diese Grundannahme führt zu weiteren Schlüssen über die Grammatikalisierung des deutschen Perfekts, die im folgenden Abschnitt dargestellt werden.

\section{Modell und Analyse}

5.1. Haben als Vollverb, Funktionsverb und Auxiliar

Die stufenweise diachrone Desemantisierung in mehreren Reanalyseschritten führte zu verschiedenen Funktionen des Verbs haben. Sie ist dank des frequenten Gebrauchs der entstandenen Konstruktionen im aktuellen deutschen Standard synchron nachvollziehbar.
a. Wir haben lauter unerledigte Probleme.
b. Wir haben alles fertig und erledigt.
(haben als Vollverb, attributives PII)
(haben als Funktionsverb) ${ }^{19}$
c. Wir haben alles erledigt. (periphrastisches Perfekt)

Im Ahd. wurde haben zunächst zu einer Art Funktionsverb reinterpretiert, indem aus Sätzen mit nachgestellten Attributen oder freien Prädi-

19. Polenz (1987) spricht hier vom Beitrag von Hilfs- und Modalverben zu partizipialen Verbgefügen; wir schlagen vor, den Begriff Funktionsverb auf alle solche Verben in partizipialen Verbgefügen auszudehnen, die nicht allein zur periphrastischen Flexion zu zählen sind. Hierbei ist aber u. E. der Gebrauch von haben in Gerundivkonstruktionen zu den grammatikalisierten Formen zu zählen:

(i) Wir haben noch einiges zu erledigen. 
kativen komplexe Prädikate reanalysiert wurden. Was aber erst das periphrastische Perfekt entstehen ließ, war die Reanalyse von perfektisch interpretierten Prädikatkomplexen zur Perfektperiphrase bei gleichzeitiger Rekategorisierung des Verbs haben zu einem Flexionselement. Diese kann auch innerhalb eines Verbalkomplexes durch die Least Effort Strategy (vgl. (22) oben) erklärt werden, wenn man eine synkretische Phrase T/VP zu Grunde legt, die von einem komplexen Kopf T/V projiziert wird (vgl. Reuland \& Kosmeijer 1993), indem die Merkmale beider Köpfe V und $\mathrm{T}$ an die dominierenden Knoten vererbt werden (Öhl 2003: 123 ff.). ${ }^{20}$ Strukturell kann dies z. B. als Adjunktion des VK, der die lexikalischen Merkmale wie die der Argumentstruktur trägt, an den Kopf $\mathrm{T}$, der die Flexionsmerkmale beherbergt, dargestellt werden:

(43)

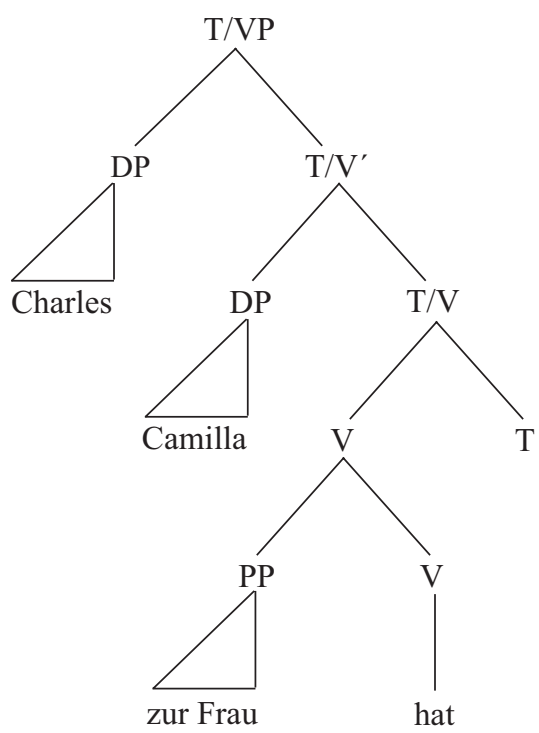

20. Wir verweisen auf Haiders (1993, 2000a) und neuerdings auch Sternefelds (2006: $507 \mathrm{ff}$.) erschöpfende Argumentation gegen eine separate Projektion einer IP in der deutschen Satzstruktur. Zugegebenermaßen ist dieses Modell nicht konform mit den gängigen minimalistischen Annahmen über syntaktische Universalien. Wir weisen jedoch darauf hin, dass die deutsche Satzstruktur bislang weder durch kopfinitiale noch durch kopffinale Flexionsphrasen befriedigend erklärt werden konnte. Die Annahme eines parametrisierten synkretischen Kopfes mit den Merkmalen $\mathrm{T}$ und $\mathrm{V}$ erlaubt uns nicht nur, dieses Problem zu umgehen, sondern auch, die Entstehung periphrastischer Flexion in OVSprachen im Rahmen eines Ökonomiemodells zu erklären. Ähnliches wurde für funktionale Phrasen z. B. von Giorgi \& Pianesi (1997) vorgeschlagen; vgl. auch Bayer (2004: 78). 
Die Grammatikalisierungsschritte sind im Folgenden anhand nhd. Beispiele demonstriert (zur Verdeutlichung in (c) auch mit halten):
a. Das Zebra hat vier Hufe, in der Regel gewetzt(e).
(nachgestelltes Attribut)
b. $\left[\mathrm{PRO}_{\mathrm{i}} \text { gewetzt }\right]^{21}$ hat seine Hufe $\mathrm{i}_{\mathrm{i}}$ das Zebra, [PRO beschla- gen] liegen sie in der Regel nur beim Hauspferd vor.
(freies Prädikativ)
c. dass ein Zebra seine Hufe immer [T/v [v gewetzt hält/hat]]
d. dass das Zebra seine Hufe [T/v gewetzt hat]
(periphrastisches Perfekt)

Die Struktur der Perfektperiphrase ist ökonomischer zu generieren als die des Satzes mit lexikalischem haben als Kopf, da in ersterer haben $\mathrm{T}$ lexikalisiert, während in letzterem die synkretische Projektion von Flexionsmerkmalen zusätzlich zum lexikalischen Kopf des Gefüges notwendig ist.
a. dass ${ }_{\mathrm{T} / \mathrm{VP}}$ das Zebra ${ }_{\mathrm{T} / \mathrm{V}^{\prime}}$ seine Hufe ${ }_{\mathrm{T} / \mathrm{V}^{*}}$ immer ${ }_{\mathrm{T} / \mathrm{V}}[\mathrm{V}$ ge- wetzt hat $\left.\left.\left.{ }_{\mathrm{V}}\right] \mathrm{T}\right] \mathrm{]}\right]$
b. dass $\left[_{\mathrm{T} / \mathrm{VP}}\right.$ das Zebra immer $\left[\mathrm{T} / \mathrm{V}^{\prime}\right.$ seine Hufe $[\mathrm{T} / \mathrm{V}$ gewetzt hat $\left.\left._{\mathrm{T}}\right] \mathrm{]}\right]$
(per. Perf.)

Die beiden für die Entstehung der Perfektkonstruktion wesentlichen Reanalysen sind in den folgenden Diagrammen expliziert. Haben wurde zunächst zum Funktionsverb in komplexen Prädikaten reinterpretiert, wo ein als kongruenzloses Prädikativ (Supinum) reanalysiertes PII das semantische Bestimmungsglied (Determinativ) bildete, haben aber den kategoriebestimmenden verbalen Kopf.

Die Existenz einer solchen Konstruktion aber erlaubte erst die ausschlaggebende Reanalyse, bei der haben durch die Least Effort Strategy als Flexionselement rekategorisiert wurde.

Abgesehen von der entscheidenden Rolle der schrittweisen Konventionalisierung der Verwendung von Prädikativkonstruktionen sind die Gradualität und das semantische Verblassen, beide Charakteristika der Grammatikalisierung, also u. a. durch die sukzessiven Reanalyseschritte zu begründen: Haben hat dadurch seine possessive Semantik in definierbaren Schritten verloren.

21. Zugegebenermaßen ist der Unterschied zwischen den Prädikationen mit haben in (b) und (c) diffus bzw. keine klare Abgrenzung erkennbar. Möglicherweise existiert im Nhd. nur noch der Typus in (c) eindeutig. Typ (b) ist aber, wie oben gezeigt, für das Ahd. belegt. 
(46)

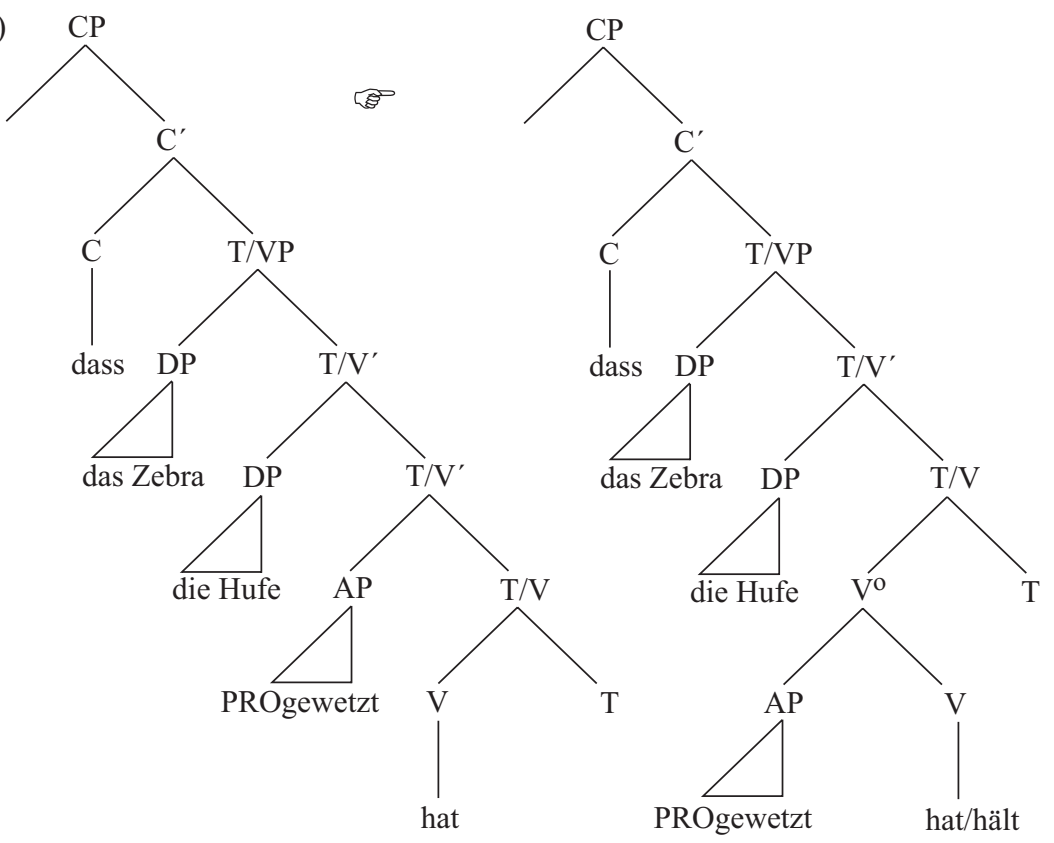

(47)

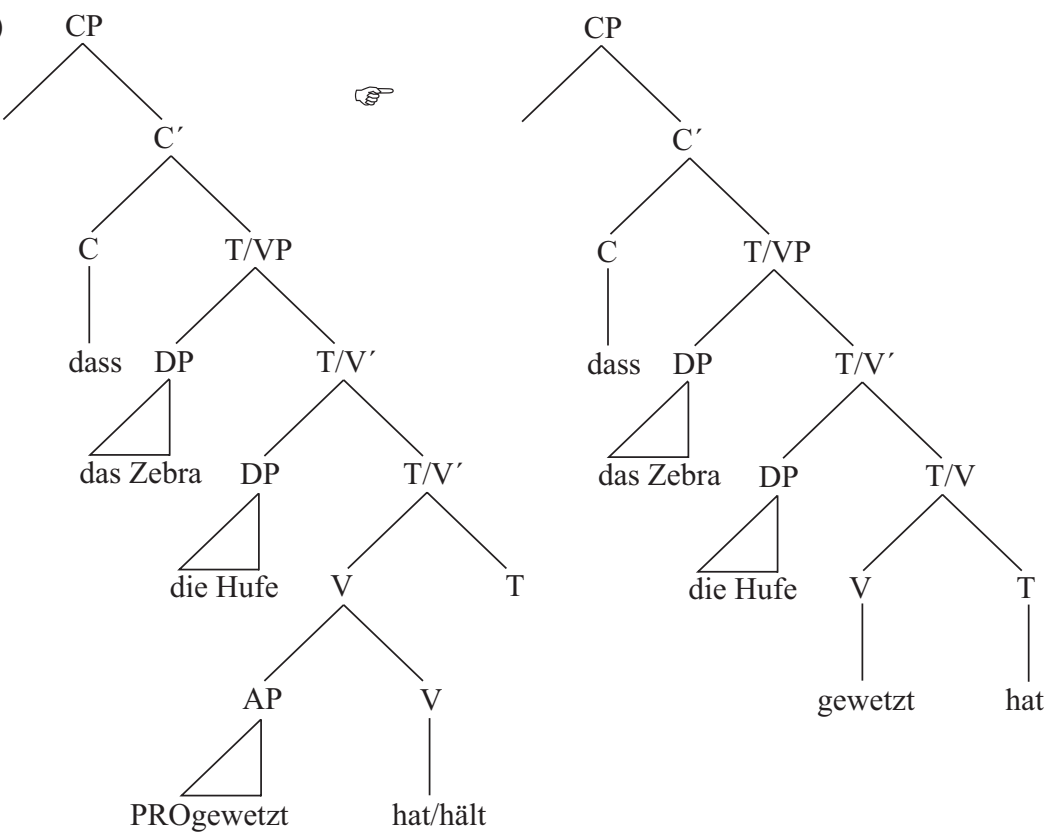




\subsection{Von implizit perfektischen Konstruktionen zum} periphrastischen Perfekt

Durch diese Reanalysen schufen die Sprachlerner jedoch nicht nur eine ökonomischere syntaktische Struktur, sondern auch die syntaktische Markierung des Perfekts durch einen neuen, periphrastischen Flexionstyp.

Wie in erläutert, ist die generelle Frage der diachronen Linguistik jene nach den Bedingungen, die für einen bestimmten Wandel vorliegen müssen (cf. Coseriu 1975). Dies betrifft die Restriktionen durch das vorhandene grammatische System, sowie das Inventar von sprachlichen Ausdrücken, die für die Erfüllung neuer Funktionen rekrutiert werden können. Die sprachlichen Rahmenbedingungen für die Grammatikalisierung des Perfektauxiliars waren im Ahd. durch die Existenz eines perfektiven Partizips gegeben, das u. a. zusammen mit haben als Objektsprädikativ verwendet wurde. Die früheren ahd. Quellen zeigen klar, dass aspektuell interpretierbare Konstruktionen mit haben + PII zunächst auf Kontexte beschränkt waren, in denen mit dem PII über ein direktes Objekt von haben sekundär prädiziert wurde (s.o. 2.1).

iogiuuelih thie thar gisihit uuîb sie zi gêronne iu habet jeglicher $R E L$ da ansieht Weib sie zu begehren schon hat sia forlegana in sinemo herzen sie geschändete in seinem Herzen

,Wer eine Frau ansieht, um sie zu begehren, hat sie schon geschändet (freies Prädikativ) in seinem Herzen. $\quad$ (Tat 28,1)

Die perfektive Semantik des PII bewirkte, dass komplexe Prädikate mit haben perfektisch interpretiert werden konnten, was bereits ab dem 9 . Jh. von den ahd. Literaten zur Darstellung von Aspekt genutzt wurde. Es gibt jedoch zunächst keine intransitiven oder ditransitiven Verben in solchen Konstruktionen, weshalb man noch nicht von einem analytischen Flexionssystem ausgehen sollte. Im Schrifttum Otfrieds finden sich 51 Belege von temporal interpretierbaren Prädikativkonstruktionen, jedoch großenteils mit kongruierendem PII, und alle mit zumindest implizitem direktem Objekt.

sie eigun ${ }^{22}$ mir ginomanan liabon druhtin minan sie haben mir genommenen lieben Herren meinen (Otfr V 7,29)

22. Eigan ,besitzen“ wird im früheren Ahd. durchgängig als Supppletivform von haben im Plural Präsens verwendet. 
Dass der Kontext aus heutiger Sicht für ein Prädikativum teils atypisch erscheint, kann nicht als Argument für eine bereits vollzogene Auxiliarisierung von haben gelten. Macht man zudem das Zugeständnis, dass die Ahd.-Sprecher den Gebrauch sekundärer PII-Prädikate als Aspektmarkierer möglicherweise bereits weitgehend konventionalisiert hatten, kann man hier von einer idiomatischen perfektischen Prädikativkonstruktion sprechen, die bis zu einem gewissen Grade sogar ohne explizites Objekt gebraucht werden konnte.
a. ir den christanum namun intfangan eigut
ihr den Christen Namen empfangen habt/besitzt (Exh. 9,5)
b. denne der paldet, der gipuazzit hapet
dann der erstarkt der gebüßt hat
(Muspilli 99)
,Dann erstarkt derjenige, der (etwas) gebüßt(es) hat.'

Wir schließen uns der Ansicht von Dik (1987: $77 \mathrm{ff}$.) an, dass periphrastische Formen aus komplexen aspektuellen Ausdrücken wie in (48) entstehen, wenn diese von Sprachlernern so weit übergeneralisiert werden, dass an die Stelle der kompositionell begründeten aspektuellen Distinktionen ein operationales Tempussystem tritt. ${ }^{23}$ Auf dem Weg dahin wurden im Ahd. zunächst aspektindizierende Prädikativkonstruktionen gebildet, deren Gebrauch konventionalisiert und auch auf für die sekundäre Prädikation atypische Kontexte (vgl. hierzu Diewald 2002; Heine 2002) ausgeweitet wurde.

Aus dieser Sicht ist es unwahrscheinlich, dass allein strukturelle Vereinfachung (bzw. derivationelle Ökonomie) zu diesen Reanalysen geführt haben kann, wie es von Roberts (1993) oder Roberts \& Roussou (2003) für das frz. Futur vorgeschlagen wurde. Die Tatsache, dass offensichtlich die aspektuelle Interpretation von Verbalkomplexen regularisiert wurde, spricht für eine semantisch motivierte Reinterpretation, was außerdem Indiz für die oben vorgeschlagene Maxime kognitiver Ökonomie ist. An die Stelle des performanzbasierten kreativen Gebrauchs eines Konstruktionstyps zur Darstellung perfektischen Aspekts trat der reguläre Gebrauch. Dies stellte eine Ökonomisierung des Sprachsystems dar, die je-

23. Vgl. hierzu auch die kompositionelle Analyse des Perfekts von Grewendorf (1995). Musan (2002) vertritt dagegen die Ansicht, dass im Nhd. sowohl Tempus- als auch Aspektoperatoren im Flexionssystem zu lokalisieren sind. Die Morphologie des Partizips spielt dagegen eine untergeordnete Rolle. Dies steht im Einklang mit der mit hier vertretenen Analyse der Rekategorisierung von haben als Flexionselement, das einen Kopf Asp bzw. einen Tempuskopf lexikalisiert, der die entsprechenden funktionalen Merkmale trägt. Eine kompositionelle Analyse ist demnach nur für die ursprünglichen aspektuellen Prädikativkonstruktionen zutreffend. 
doch nur durch dessen parametrische Restrukturierung möglich war, die fortan die regelbasierte Lexikalisierung dieses Aspektmerkmals erlaubte.

Erst als haben als Auxiliar bereits rekategorisiert war, konnte das haben-Perfekt auch mit intransitiven Verben gebildet werden (vgl. Leiss 1992: 271 ff.). Auch wenn eine solche kontextuell stark eingeschränkte Prädikativkonstruktion zur Darstellung von aspektuellen Verhältnissen dienen kann, sollte erst dann von einem verbalen Flexionstyp gesprochen werden, wenn damit sämtliche für die Temporalflexion in Frage kommenden Verbtypen flektiert werden können, wenn sie also paradigmatisch ist. PII + Auxiliar als periphrastisches Perfekt gibt es somit weder im ahd. Tatian noch bei Otfried. Erst 150 Jahre später, in den ahd. Quellen ab Notker, bilden intransitive und ditransitive Verben ein PII, und das nur in Verbindung mit dem Auxiliar. Ab diesem Moment kann mit Sicherheit gesagt werden, dass das PII kein prädikatives Attribut zum direkten Objekt darstellt. Es bildet auch nicht den ergänzenden Teil in einem komplexen Prädikat (wie bei lieb haben o. ä.), sondern ist ein autonomes Prädikat, das mit Hilfe des Auxiliars, das die Flexionsmerkmale lexikalisiert, analytisch flektiert wird.
a. so habet er gelogen
,So hat er gelogen.'
b. tar habet si imo geantwurtet sinero frago
,Da hat sie ihm auf seine Frage geantwortet.

(Notk I, 544; 29)

(Notk I,284;26)

Der grammatische Wandel, der letztlich die Systeminnovation im Sinne Coserius (1975) darstellt, fand abrupt statt, als die Rekategorisierung zum T-Element stattfand. Diese war vollzogen, als in den Quellen haben als Auxiliar mit allen Verbtypen, außer denen, die ihr Perfekt mit sein bilden, regulär verwendet wurde. Das haben-Perfekt ist also durch das Zusammenwirken von performanzbasiertem und parametrischem Wandel entstanden, oder, in Coserius Worten: durch mehrere Normwandel, die zu Systemwandeln führten.

\subsection{Haben oder sein}

Einige grammatische Merkmale von haben persistierten ${ }^{24}$ augenscheinlich auch nach der Auxiliarisierung, und dies bis in das heutige Perfektsystem. Hierzu gehören gewisse Eigenschaften des Selektionsrahmens. Im Gegensatz zum Englischen, wo have vollständig als Perfektauxiliar

24. Zum Terminus Persistenz vgl. Hopper \& Traugott (1993: 3). 
für sämtliche Verbtypen grammatikalisiert worden ist (s. hierzu Zimmermann 1978), bildet bekanntlich im Deutschen (wie auch im Niederländischen, Französischen und Italienischen) eine definierbare Gruppe von Verben ihr Perfekt nicht mit haben, sondern mit sein.
a. Der Sturm hat den Baum gefällt.
b. Der Baum ist im Sturm gefallen.

Hierfür wurden verschiedene grammatiktheoretische Erklärungen vorgeschlagen, die zum einen auf der syntaktischen Argumentstruktur beruhen (vgl. Haider 1984, 2000b; Burzio 1986; Haider \& Rindler-Schjerve 1987), zum anderen auf semantischen Konzepten wie Agentivität und Aktionsart (Abraham 2000).

Die Ansicht, dass die Auxiliarwahl von der Aktionsart des Verbs abhängig ist, wird bereits von Behaghel (1899) vertreten, der feststellt, dass die Verwendung von sein „von gewissen Störungen abgesehen“ (Behaghel 1899: 68) auf perfektive Verben beschränkt sei. Er führt als Beleg mhd. Minimalpaare polysemer Verben an, deren perfektive Lesart die Auxiliarselektion von sein bewirkt.
a. ich hân geswigen
,ich habe den Mund gehalten
b. ich bin geswigen
,ich bin verstummt

Intransitiven Verben, die ihr Perfekt nicht mit sein bilden, ist gemeinsam, dass ihr PII nicht attributiv verwendet werden kann:
a. *der geschlafene Student
b. *der genieste Patient

Diese PII konnten vor der Einführung des haben-Perfekts überhaupt nicht gebildet werden, sondern sind, wie schon Behaghel (1899) feststellt, „erst mit der perfectumschreibung und durch sie ins leben getreten“ (Behaghel 1899: 69). Es steht nun zur Diskussion, was die Sprecher des Deutschen veranlasste, für alle Verben außer denen des Typs (52b), in ihrem neuen Flektionssystem als Auxiliar ausschließlich haben zu verwenden, und nicht etwa sein über die intransitiven Verben hinweg zu generalisieren.

(55) a. *Die Frau ist aufgelacht.

b. *Der Mann ist gelogen.

c. *Das Kind ist geschlafen. 
Eine rein formale Erklärung dieses Umstands bietet die generative Syntax, wo aufgrund von Eigenschaften der Argumentstruktur innerhalb der Intransitiva die Untergruppe der ergativen bzw. unakkusativischen Verben eingegrenzt wird. Die beiden Termini beruhen auf zwei korrelierenden Charakteristika dieser Verben. Ihre Subjekte verhalten sich in einigen Belangen wie die Objekte transitiver Verben. So ist das PII eines transitiven Verbs attributiv nur auf sein Objekt zu beziehen, bei diesen Verben jedoch auf das Subjekt.

$$
\begin{array}{ll}
\text { a. } & \text { der gefällte Baum ( } \mathrm{V}^{\text {tr }} \text { fällen) } \\
\text { b. } & \text { der gefallene Baum ( }\left(\mathrm{V}^{\text {intr }}\right. \text { fallen) }
\end{array}
$$

Analog zur sprachtypologischen Klasse der Ergativsprachen, wo sich, grob generalisiert, die Subjekte wie Objekte in Nominativ/Akkusativsprachen verhalten, wurden diese Verben bereits von Anderson (1968) ergative Verben genannt. In der neueren generativen Literatur wird der neutralere Begriff unakkusativisch bevorzugt, der von der typischen Eigenschaft dieser Verben abgeleitet ist, niemals an ein Objekt Akkusativ zu vergeben, auch nicht an so genannte ,kognate Objekte', die bei vielen anderen Intransitiven möglich sind (vgl. Perlmutter 1978):

$$
\begin{aligned}
& \text { a. Er schläft einen tiefen Schlaf. } \\
& \text { b. } \quad \text { Er fällt einen tiefen Fall. }
\end{aligned}
$$

Diese Eigenschaft lässt sich im generativen Modell dadurch erklären, dass die Subjekte unakkusativischer Verben eigentlich syntaktisch abgeleitete direkte Objekte sind (vgl. auch Grewendorf 1989): Durch die syntaktische Derivation erhält das konzeptuelle ,direkte Objekt' eines Verbs, dessen lexikalisch-konzeptuelle Struktur kein für die Subjektsfunktion designiertes Argument enthält, die syntaktische Subjektsfunktion. Nach Haider \& Rindler-Schjerve (1987) weisen die Auxiliare in Sprachen mit variierender Auxiliarselektion, vereinfacht ausgedrückt, eine nicht auf semantische Rollen festgelegte Argumentstruktur auf, die mit dem $\Theta-$ Raster des Hauptverbs kompatibel sein muss. Da das Auxiliar sein (wie auch die Kopula, von der es diachron abgeleitet ist) konzeptuell kein Subjekt lizenziert, ${ }^{25}$ kann es nur Prädikate ohne ein für die Subjektsfunktion designiertes Argument selegieren (vgl. Haider 1984) Verben mit designiertem Argument werden von haben selegiert, dessen Argumentstruktur ein solches lizenziert. Im Sinne eines Grammatikalisierungsmo-

25. Subjekte von Prädikativsätzen sind syntaktisch vom (tiefenstrukturell wie logisch) internen Argument der Prädikation abgeleitet. 
dells könnte der Erhalt einer (nicht semantisch definierten) Argumentstruktur also als Persistenzphänomen klassifiziert werden.

Shannon (1990) wendet sich gegen diese Arten der syntaktischen Erklärung: ,[...] Unaccusativity [...] I do not believe is a real or even coherent concept [...].“ (Shannon 1990: 470). Er argumentiert, dass sich die Wahl in den meisten Fällen besser durch Affinität zu prototypischen Eigenschaften von Verben wie Intransitivität, Non-Agentivität und Mutativität erklären ließe. Letztere Eigenschaft komme Ereignissen zu, bei denen „ein Aktant seinen Zustand oder seine Position verändert“" (Shannon 1990: 469). Das Gewicht weiterer prototypischer Eigenschaften variiere sprachspezifisch. Shannon führt folgende Argumente für seine Analyse an:

1. Bei Bewegungsverben entscheide allein die Mutativität, ob haben oder sein selegiert werde (vgl. Deutsch: Ich habe gerudert - Ich bin zur anderen Seite gerudert).

2. Die Inkorporation von Objekten (nhd. Autofahren) und Klitisierung von Reflexiva im Romanischen löse Detransivierung aus und erlaube deswegen Selegierung von sein als Perfektauxiliar.

3. Sein selegieren im Frz. nur wenige, im Dt. und Ndl. viele, im Ital. sehr viele Verben als Perfektauxiliar. Also sei bezüglich der Prototypikalität letzteres am wenigsten, ersteres am stärksten selektiv.

Er stellt die Hypothese auf, dass in den hier relevanten Grammatikalisierungsprozessen die Auxiliare in allen Sprachen zunächst mit den prototypischsten Vertretern von Prädikaten verwendet werden und sich dann in sprachspezifischer Variation über das gesamte Verbsystem ausbreiten: „The claim is that there are prototypes for have and be as auxiliaries, that the development begins with these and that gradual extension takes place in various directions until all verbs are covered by one of the perfect auxiliaries." (Shannon 1990: 471).

Nimmt man Shannons prototypische Kontexte für haben als Auxiliar als solche, die die aspektuelle Lesart eines funktionalisierten Vollverbs begünstigen, können Shannons Feststellungen den hier vertretenen integrativen Ansatz durchaus unterstützen. Es ist nachvollziehbar, dass vor der Rekategorisierung, nämlich während des Prozesses der Konventionalisierung aspektuell interpretierbarer Prädikativkonstruktionen, die Restriktion ihrer Verwendung zurückging, wobei jedoch nicht-regelbasierter Einsatz (wie z. B. die Bildung eines nicht-prädikativ verwendbaren PII eines Verbs wie schlafen) weiterhin ausgeschlossen gewesen sein muss. Solche Prädikativkonstruktionen existierten im Ahd. sowohl mit haben als auch mit sein. Voraussetzung für beide Konstruktionen war, dass das PII prädikativ auf ein logisches Objekt bezogen wurde, das aber im 
zweiten Fall einem syntaktischen Subjekt entsprach. Beide Strukturen boten das Potential für die Rekategorisierung des flektierten Teils des Prädikats: zum einen haben, zum anderen sein.

Dik (1987) betrachtet die Auxiliarisierung der Kopula als reine Expansion auf eine neue grammatische Funktion, die keine Desemantisierung involviert. Sie beginnt mit ,innovativen aspektuellen Formen, die später als Tempus oder diathetisch reinterpretiert werden können" (Dik 1987: 80). Diese Reinterpretation ist in seiner Sicht semantische Vereinfachung, die darauf beruht, dass Sprecher oder Sprachlerner die komplexe Relation zwischen einem Referenten und einem resultativen Prädikat, das aus einem Ereignis folgt, dahingehend ersetzen, dass fortan die direkte Relation zwischen dem Referenten und dem Ereignis ausgedrückt wird (Dik 1987: 75 ff.).

Caesar victus est.

Caesar besiegt-wordener ist

,Caesar ist besiegt worden.

(vgl. Dik 1987: 69)

Dieser Effekt würde in einem generativen Ansatz direkt aus dem Umstand folgen, dass die Rekategorisierung der Kopula (die nach gängigen Annahmen von der Kategorie V ist) als funktionaler Kopf (Asp oder T) die Reanalyse des Prädikativums im Ausgangssatzes zum verbalen Kopf bedingt, sodass dieses nun über das Ereignis prädiziert. Wir stimmen mit Dik (1987) überein, dass sowohl die Kopula als auch das Auxiliar semantisch leer sind. Der formale Unterschied besteht darin, dass der Lexikoneintrag des Auxiliars die Tempusmerkmale beinhaltet, mit denen die Kopula syntaktisch assoziiert werden muss. Wie auch bei der Erklärung der Auxiliarisierung von haben teilen wir jedoch Diks Ansicht, dass vor der Reinterpretation zur Perfektperiphrase die Konventionalisierung der aspektuellen Prädikativkonstruktion stand.

Da Kopulakonstruktionen kein designiertes Argument besitzen, erklärt es sich u. E. von selbst, dass zumindest zunächst nur unakkusativische Verben mit sein konstruiert wurden. Aus generativer Sicht wäre es jedoch völlig verwunderlich, wenn sich die Verwendung des Auxiliars sein, das die Argumentstruktur der Kopula ererbt hat, auf Verben mit externem Argument ausgeweitet hätte.

Wenn sein mit Bewegungsverben verwendet wird, sollte dies daher rühren, dass ein overtes direktes Objekt deswegen blockiert ist, weil ihr Subjekt von einem Argument in Komplementposition abgeleitet ist. Dies wird zumindest indirekt dadurch bestätigt, dass eben dann, wenn ein solches Verb haben zum Auxiliar hat, ein direktes Objekt möglich (oder sogar notwendig) ist. 
(59) a. Ich habe (ein Boot) gerudert.

b. Er hat*(ein schnelles Auto) gefahren.

In diesem Fall wird die lexikalisch-konzeptuelle Struktur von Bewegungsverben um ein designiertes Argument erweitert. Sie wird nicht etwa im umgekehrten Falle der Abwesenheit eines Objekts reduziert. Die veränderte Auxiliarwahl bei Inkorporation von Objekten nur dieser Verben zeigt nämlich, dass der Selektionsrahmen des Basisverbs die relevante Restriktion darstellt. Im Gegensatz zur Inkorporation an sich ist der Auxiliarwechsel nämlich alles andere als produktiv:
a. Er ist autogefahren.
b. Er hat $/ *$ ist autogewaschen.

Diese Beispiele zeigen auch, dass es schwierig ist, die Relevanz der semantischen Rollen der Argumente eines Prädikats bei der Auxiliarwahl plausibel zu machen. Das Subjekt in (a) sollte nach einer Analyse im Sinne Shannons (1990) eine non-agentive thematische Rolle tragen. Das Fahren eines Autos ist jedoch kaum mehr oder weniger agentiv als das Waschen. Das Gleiche sollte für Minimalpaare gelten wie:

(61) a. Ich habe in Stuttgart gewohnt/gelebt/gewartet.

b. Ich bin in Stuttgart gewesen/geblieben/gestanden.

(62) a. Ich bin von Stuttgart weggegangen.

b. Ich habe Stuttgart verlassen.

Eine nahe liegendere Annahme scheint zu sein, dass diese Verben gemäß rein formaler Prinzipien der Argumentstruktur lexikalisch unterschiedlich parametrisiert sind. Hierdurch lassen sich letztlich auch parametrische Unterschiede zwischen Sprachen wie Französisch und Deutsch erklären, die bei Verben identischen semantischen Konzepts unterschiedliche Auxiliare verwenden.

a. J'ai couru à l'école.

b. Ich bin zur Schule gerannt.

Wir stellen also fest, dass, solange es lediglich die Konventionalisierung der beiden Prädikativkonstruktionen zur Darstellung perfektischer Sachverhalte betraf, sehr wohl eine Ausweitung im Sinne Shannons (1990) auf eine größere Anzahl von Ereigniskontexten geben konnte. Da es sich jedoch in beiden Fällen nach wie vor um Prädikative handelte, konnten 
nur Partizipien transitiver (mit haben) bzw. unakkusativischer (mit sein) Verben verwendet werden. Nachdem haben als funktionaler Ausdruck rekategorisiert war, wurde es auch mit Verben, deren Lexikoneintrag kein Akkusativobjekt vorsah, verwendet, so lange diese ein designiertes Argument besaßen. Auf unakkusativische Verben wurde das haben-Perfekt nicht ausgeweitet, da die parallel existierende, aspektuell interpretierbare Konstruktion Kopula + PII die konvergente Grammatikalisierung und Reanalyse des sein-Perfekts der Unakkusativa begünstigte. Dies begründet die noch heute bestehende, nach Verbtypen differenzierte Auxiliarselektion diachron. Dass diese erhalten blieb, ist u. E. ein Argument dafür, dass in Sprachen wie Deutsch und Französisch bei Auxiliaren trotz Funktionalisierung auch Eigenschaften der Argumentstruktur erhalten geblieben sind bzw. persistierten.

Dass in Sprachen wie Englisch haben alleiniges Perfektauxiliar ist, muss bedeuten, dass dort dessen Argumentstruktur nicht erhalten wurde, die Grammatikalisierung also weiter fortgeschritten ist. Wir schlagen vor, dass dies genau dann der Fall ist, wenn ein ursprünglich lexikalisches Element als Lexikoneintrag für einen rein funktionalen Kopf rekategorisiert wurde, der nur noch Flexionsmerkmale (Tempus, Aspekt und Kongruenz) trägt. Das Perfektauxiliar in Sprachen mit Auxiliarvariation ist dagegen in einer tieferen Position generiert, die mit lexikalischen Merkmalen assoziiert ist. Dies mit unserem Vorschlag in Zusammenhang zu bringen, dass das Perfektauxiliar im Deutschen zusammen mit dem Prädikat im Verbalkomplex steht, würde voraussagen, dass dies auch für Sprachen wie Italienisch und Französisch gilt, was wesentlich weiter reichender Diskussion bedürfte. Es besteht jedoch die Möglichkeit, dass nur in haben/sein-Sprachen das Auxiliar als V basisgeneriert ist, während es in Sprachen wie Englisch direkt als ein funktionaler Kopf Asp in die Struktur eingefügt und im Falle der Finalität des Auxiliars von dort nach T bewegt wird.
a. Charles [те will [AspP have [vP married Camilla]]] by the end of the year.
b. Charles and Camilla [TP have [AspP $\mathrm{t}[\mathrm{VP}$ married]]] .

\section{Zusammenfassung und Schluss}

Ein analytischer Flexionstyp Partizip + Auxiliar liegt nur dann vor, wenn durch ihn alle Verben eines grammatischen Systems in ein Flexionsparadigma integriert sind. Dies ist auch dann der Fall, wenn in diesem Paradigma regelhaft für verschiedene Verbklassen unterschiedliche Auxiliare verwendet werden. Wenn aber eine Sprache wie das frühe Alt- 
hochdeutsche aspektuelle Prädikativkonstruktionen nur mit bestimmten Verben bildet, sind diese lediglich innerhalb dieses Konstruktionstyps paradigmatisch, selbst wenn die Verwendung der Konstruktion zur Aspektmarkierung in hohem Grade konventionalisiert war und die Interpretation der Perfektivität Tendenzen zur Generalisierung aufwies. Es wurde in diesem Beitrag deshalb dafür argumentiert, dass die Auxiliarisierung des Verbs haben, mit der die Konstruktion ohne einen feststellbaren graduellen Übergang auf intransitive Verben ausgeweitet wurde, einen abrupten Sprachwandel darstellte: die Reanalyse eines Verbalkomplexes mit haben als Funktionsverb zu einer Struktur, in der haben einen funktionalen Kopf lexikalisiert.

Die Gradualität des Grammatikalisierungsprozesses gründet darauf, dass zum einen eine vorausgehende Reanalyse komplexe Prädikate mit dem ursprünglichen verbum possessivum als Kopf geschaffen hatte, zum anderen die schrittweise Konventionalisierung aspektueller Prädikativkonstruktionen die Grundlage für die spätere Rekategorisierung schuf. Aufgrund dieser Wechselbeziehung parametrischer und performanzbasierter Wandel lässt sich die Grammatikalisierung des Perfektauxiliars weder mit rein formalen noch mit rein funktionalen Methoden erklären. Aus diesem Grund wurde in diesem Beitrag für ein integratives Modell argumentiert, das die relevanten Aspekte beider Arten von Wandel in der Diachronie der Perfektkonstruktion zu fassen in der Lage ist.

In Sprachen wie dem Deutschen, in denen Prädikativkonstruktionen mit unakkusativischen Verben mit der Kopula sein gebildet wurden, boten diese eine vergleichbare Basis für Konventionalisierung und Reanalyse. Das Auftauchen beider Auxiliare in einem Flexionsparadigma ist jedoch weniger als Konkurrenz denn als Suppletion zu interpretieren, da das Perfekt des jeweiligen Verbtyps wesentliche Eigenschaften (u. E. die Argumentstruktur) von der Grammatikalisierungsbasis ererbt hat. Nur in Sprachen wie dem Englischen, in denen das Auxiliar nur noch die spezifischen Flexionsmerkmale trägt, ist das Suppletiv redundant und wurde aufgrund von Ökonomieprinzipien verdrängt. Wir haben dafür plädiert, dass es dann den Kopf Asp direkt lexikalisiert, der im Gegensatz zu V nicht mit Merkmalen der Argumentstruktur assoziiert ist.

\section{Literatur}


Eggers, Hans (1964). Der althochdeutsche Isidor. Nach der Pariser Handschrift und den Monseer Fragmenten neu herausgegeben. Tübingen: Niemeyer.

Gabelentz, Hans C. von \& Julius Loebe (1843). Ulfilas. Veteris et novi testamentis versionis Gothicae cum glossario et grammatica linguae Gothicae. Leipzig: F. A. Brockhaus.

Katholische Bibelanstalt Stuttgart (1980) (Hg.). Hausbibel: Einheitsübersetzung des Alten und Neuen Testaments. Stuttgart: Katholische Bibelanstalt.

Piper, Paul ([1878] 1982) (Hg.). Otfrieds Evangelienbuch. 1. Theil: Einleitung und Text. Hildesheim: Olms.

Tax, Petrus W. (1986-90) (Hg.). Die Werke Notkers des Deutschen. 2. Auflage. Tübingen: Niemeyer.

Volz, Hans \& Heinz Blanke (Hgg.) (1973). Martin Luther: Biblia. Das ist die Ganze Heilige Schrifft Deudsch aufs new zugericht. Darmstadt: Wissenschaftliche Buchgesellschaft.

\section{Fachliteratur}

Abraham, Werner (1986). The grammar of German haben. In Linguistics across Historical and Applied Linguistics, Dieter Kastovsky \& Aleksander Szwedek (eds.), 781-797. Berlin, New York: Mouton de Gruyter.

Abraham, Werner (1990). Die Grammatikalisierung von Auxiliar- und Modalverben. In: Beiträge zur Geschichte der deutschen Sprache und Literatur 112; 200-208.

Abraham, Werner (1991). Aktionsartsemantik und Auxiliarisierung im Deutschen. In Neue Fragen der Linguistik, Elisabeth Feldbusch, Reiner Pogarell \& Cornelia Weiß (Hgg.), 125-133. Tübingen: Niemeyer.

Abraham, Werner (1992a). Event structure accounting for the emerging periphrastic tenses and the passive voice in German. In Explanation in Historical Linguistics, Garry W. Davis \& Gregory K. Iverson (eds.), 1-15. Amsterdam: Benjamins.

Abraham, Werner (1992b). Grammatikalisierung und Reanalyse: einander ausschließende oder ergänzende Begriffe? Folia Linguistica Historica 13: 7-26.

Abraham, Werner (2000). The aspect-case typology correlation: perfectivity and Burzio's generalization. In: Arguments and Case: Explaining Burzio's Generalization, Eric Reuland (ed.), 131-193. Amsterdam: Benjamins.

Abraham, Werner (2004). The grammaticalization of the infinitival preposition. Toward a theory of ,grammaticalizing reanalysis‘. Journal of Comparative Germanic Syntax 7: $111-170$.

Abraham, Werner (2006). Introduction: Passivization and typology. In Passivization and Typology: Form and Function, Werner Abraham (ed.), 1-27. Amsterdam: Benjamins.

Andersen, Henning (1973). Abductive and deductive change. Language 49: 765-793.

Anderson, John M. (1968). Ergative and nominative in English. Journal of Linguistics 4: $1-32$.

Bayer, Josef (2004). Decomposing the left periphery. Dialectal and cross-linguistic evidence. In The Syntax and Semantics of the Left Periphery, Horst Lohnstein \& Susanne Trissler (Hgg.), 59-95. Berlin, New York: Mouton de Gruyter.

Behaghel, Otto (1899). Ich habe geschlafen. Zeitschrift für Deutsche Philologie 32: 64-72.

Behaghel, Otto (1924). Deutsche Syntax. Eine geschichtliche Darstellung. Heidelberg: Winter.

Bierwisch, Manfred (1990). Verb cluster formation as a morphological process. In: Yearbook of Morphology 3, Geert Boij \& Jaap van Marle (eds.), 173-199. Dordrecht: Foris.

Braune, Wilhelm \& Hans Eggers (1987). Althochdeutsche Grammatik. 14. Auflage. Tübingen: Niemeyer.

Brinkmann, Hennig (1931). Sprachwandel und Sprachbewegungen in althochdeutscher Zeit. Jena: Frommann.

Burzio, Luigi (1986). Italian Syntax - A Government and Binding Approach. Dordrecht: Reidel. 
Chomsky, Noam \& Howard Lasnik (1993). The theory of principles and parameters. In Syntax: An International Handbook of Contemporary Research, [HSK 9/1], Joachim Jacobs, Arnim von Stechow, Wolfgang Sternefeld \& Theo Vennemann (eds.), 506-569. Berlin, New York: Mouton de Gruyter.

Chung, Sandra (1977). On the gradual nature of syntactic change. In: Mechanisms of Syntactic Change, Charles N. Li (ed.), 3-56. Austin, London: University of Texas Press.

Coseriu, Eugenio (1974). Synchronie, Diachronie und Geschichte. Das Problem des Sprachwandels. München: Fink

Coseriu, Eugenio (1975). Synchronie, Diachronie und Typologie. In: Sprachwandel. Reader zur Diachronischen Sprachwissenschaft, Dieter Cherubim (Hg.), 135-149. Berlin, New York: Mouton de Gruyter.

Dal, Ingerid (1966). Kurze deutsche Syntax auf historischer Grundlage. 3. Auflage. Tübingen: Niemeyer.

Diewald, Gabriele (2002). A model for relevant types of contexts in grammaticalization. In: New Reflections on Grammaticalization, Ilse Wischer \& Gabriele Diewald (eds.) (2002), 103-130. Amsterdam: Benjamins.

Dik, Simon C. (1987). Copula auxiliarization: how and why? In: Historical Development of Auxiliaries, Martin Harris \& Paolo Ramat (eds.), 53-84. Berlin, New York: Mouton de Gruyter.

Erben, Johannes (1976). Sprachgeschichte als Systemgeschichte. In: Sprachwandel und Sprachgeschichtsschreibung, Hugo Moser (Hg.), 7-23. Düsseldorf: Schwann.

Fukui, Naoki (2002). Phrase Structure. In The Handbook of Contemporary Syntactic Theory, Mark Baltin \& Chris Collins (eds.), 374-406. Oxford: Blackwell.

Gelderen, Elly van (1993). Reanalysis as a response to grammaticalization. GAGL 36, $169-83$.

Gelderen, Elly van (1996). The Rise of Functional Categories. Amsterdam: Benjamins.

Gelderen, Elly van (2004). Grammaticalisation as Economy. Amsterdam: Benjamins.

Giorgi, Alessandra \& Fabio Pianesi (1997). Tense and Aspect. From Semantics to Morphosyntax. Oxford: Oxford University Press.

Grandgent, Charles H. ([1934] 1962). An Introduction to Vulgar Latin. New York: Hafner.

Grewendorf, Günther (1989). Ergativity in German. Dordrecht: Foris.

Grewendorf, Günther (1995). Präsens und Perfekt im Deutschen. Zeitschrift für Sprachwissenschaft: $72-90$.

Grimm, Jakob: Deutsche Grammatik. Hg. v. Gustav Roethe und Eduard Schröder (1967). Bd. IV: Syntax. Hildesheim: Olms.

Grønvik, Ottar (1986). Über den Ursprung der aktiven Perfekt und Plusquamperfektkonstruktion im Deutschen. Oslo: Solum.

Haider, Hubert (1984). Was zu haben ist und was zu sein hat. Bemerkungen zum Infinitiv. Papiere zur Linguistik 30: 23-36.

Haider, Hubert (1993). Deutsche Syntax - generativ. Tübingen: Narr

Haider, Hubert (1994). Fakultativ kohärente Infinitivkonstruktionen im Deutschen. In: Zur Satzwertigkeit von Infinitiven und Small Clauses, Anita Steube \& Gerhild Zybatow (Hgg.), 75-106. Tübingen: Niemeyer.

Haider, Hubert (2000a). Branching and discharge. In Lexical Specification and Insertion, Peter Coopmans, Martin Everaert \& Jane B. Grimshaw (eds.), 135-164. Amsterdam: Benjamins.

Haider, Hubert (2000b). The License to license: licensing of structural case plus economy yields Burzio's Generalization. In: Arguments and Case: Explaining Burzio's Generalization, Eric Reuland (ed.), 31-55. Amsterdam: Benjamins.

Haider, Hubert \& Rositta Rindler-Schjerve (1987). The parameter of auxiliary selection. Italian-German contrasts. Linguistics 25: 1029-1055.

Harris, Martin \& Paolo Ramat (1987) (eds.). Historical Development of Auxiliaries. Berlin, New York: Mouton de Gruyter. 
Hartmann, Katharina (2001). Right Node Raising and Gapping. Interface Conditions on Prosodic Deletion. Amsterdam: Benjamins.

Haspelmath, Martin (1994). Functional categories, X-bar theory, and grammaticalization theory. Sprachtypologie und Universalienforschung 47: 3-15.

Haspelmath, Martin (1998). Does grammaticalization need reanalysis? Studies in Language 22: $31-352$.

Heine, Bernd (1993). Auxiliaries. Cognitive Forces and Grammaticalization. Oxford: Oxford University Press.

Heine, Bernd (2002). On the role of context in grammaticalization. In: New Reflections on Grammaticalization. Ilse Wischer \& Gabriele Diewald (eds.), 83-102. Amsterdam: Benjamins.

Hopper, Paul \& Elisabeth Traugott (1993). Grammaticalization. Cambridge: Cambridge University Press.

Jacob, Daniel (1998). Transitivität, Diathese und Perfekt: zur Entstehung der romanischen haben-Periphrasen. In Transitivität und Diathese in romanischen Sprachen, Hans Geisler \& Daniel Jacob (Hgg.), 105-126. Tübingen: Niemeyer.

Kayne, Richard S. (1994). The Antisymmetry of Syntax. Cambridge MA: MIT Press.

Kiparsky, Paul (1975). Sprachuniversalien und Sprachwandel. In: Sprachwandel. Reader zur Diachronischen Sprachwissenschaft, Dieter Cherubim (Hg.), 237-276. Berlin, New York: Mouton de Gruyter.

Kuryłowicz, Jerzy (1965). The evolution of grammatical categories. Diogenes 51: 55-71.

Latzel, Sigbert (1977). Haben + Partizip und ähnliche Fügungen. Deutsche Sprache 5: $289-312$.

Langacker, Ronald W. (1977). Syntactic reanalysis. In: Mechanisms of Syntactic Change, Charles N. Li (ed.), 57-139. Austin, London: University of Texas Press.

Lehmann, Christian ([1982] 1995). Thoughts on Grammaticalization. München: Lincom Europa (ursprünglich erschienen als: Thoughts on Grammaticalization: A programmatic sketch. Vol. 1. Universität zu Köln: Arbeiten des Kölner Universalienprojekts 49).

Lehmann, Winfried P. (1973). A structural principle of language and its implications. Language 49: 47-66

Leiss, Elisabeth (1992). Die Verbalkategorien des Deutschen: Ein Beitrag zur Theorie der Kategorisierung. Berlin, New York: Mouton de Gruyter.

Lightfoot, David (1979). Principles of Diachronic Syntax. Cambridge: Cambridge University Press.

Lightfoot, David (1991). How to Set Parameters. Arguments from Language Change. Cambridge MA: MIT Press.

Martinet, André ([1955] 1981). Sprachökonomie und Lautwandel. Eine Abhandlung über die diachronische Phonologie. Stuttgart: Klett-Cotta.

McMahon, April (1993). Understanding Language Change. Oxford: Blackwell.

Meillet, André (1912). L'évolution des formes grammaticales. In Linguistique Historique et Linguistique Génerale, André Meillet (Hg.), 130-148. Paris: Champion.

Morris, Richard L. (1988). The rise of periphrastic perfect tenses in German: the case against Latin influence. In Stafcraft: Studies in Germanic Linguistics, Elmer H. Antonsen \& Hans Henrich Hock (eds.), 161-167. Amsterdam: Benjamins.

Müller, Stefan (2002). Complex Predicates: Verbal complexes, Resultative Constructions, and Particle Verbs in German. Stanford: CSLI Publications.

Musan, Renate (2002). The GermanPperfect: Its Semantic Composition and its Interactions with Temporal Adverbials. Dordrecht: Kluwer.

Öhl, Peter (2003). Economical computation of structural descriptions in natural language. Dissertation, Universität Stuttgart. (Online-Publikation: http://elib.uni-stuttgart.de/ opus/volltexte/2003/1419/pdf/DissertationOehl.pdf )

Öhl, Peter (2006). Über Sinn und Nutzen einer Generativen Grammatiktheorie. In Sprache und Sprachen im Mitteleuropäischen Raum. Vorträge der Internationalen Linguistik-Tage 
Trnava 2005, Kozmová, Ružena (Hrsg.), 229-243. Trnava: Univerzita sv. Cyrila a Metoda.

Öhl, Peter (2008). Althochdeutsche Literatur im Rahmen einer Theorie der Translation. In Linguistica et Res Cotidianae, Iwona Bartoszewicz, Joanna Szczęk \& Artur Tworek (Hgg.), 431-444. Beihefte zum Orbis Linguarum 68. Wroclaw, Dresden: Neisse Verlag.

Öhl, Peter \& Agnes Korn (2006). Performative und parametrische Wandel in der linken Satzperipherie des Persischen. Der Subordinationsmarkierer ke und die Interrogativpartikel āyā. Die Sprache 46: 137-202.

Oubouzar, Erika (1975). Über die Ausbildung der zusammengesetzten Verbformen im deutschen Verbalsystem. Beiträge zur Geschichte der deutschen Sprache und Literatur 95: 5-96.

Perlmutter, David M. (1978). Impersonal passive and the unaccusative hypothesis. Proceedings of the Fourth Annual Meeting of the Berkeley Linguistics Society (BLS 4), 157-189. Berkley CA: University Press.

Polenz, Peter von (1987). Funktionsverben, Funktionsverbgefüge und Verwandtes. Vorschläge zur satzsemantischen Lexikographie. Zeitschrift für Germanistische Linguistik 15: $169-189$.

Radford, Andrew, Martin Atkinson, David Britain, Harald Clahsen \& Andrew Spencer (2006): Linguistics. An Introduction. Cambridge: Cambridge University Press.

Ramat, Paolo (1982). Ein Beispiel von Reanalysis, typologisch betrachtet. Folia Linguistica 16: $365-383$.

Reuland, Eric \& Wim Kosmeijer (1993). Projecting inflected verbs. In The Parametrization of Universal Grammar, Gisbert Fanselow (ed.), 37-71. Amsterdam: Benjamins.

Roberts, Ian (1993). A formal account of grammaticalization in the history of Romance futures. Folia Linguistica Historica 13: 219-258.

Roberts, Ian \& Anna Roussou (2003). Syntactic Change. A Minimalist Approach to Grammaticalization. Cambridge: Cambridge University Press.

Shannon, Thomas F. (1990). The unaccusative hypothesis and the history of the perfect auxiliary in Germanic and Romance. In Historical Linguistics 1987, Henning Andersen \& K. Koerner (eds.), 461-488. Amsterdam: Benjamins.

Sternefeld, Wolfgang (2006). Syntax. Eine morphologisch motivierte generative Beschreibung des Deutschen. 2 Bände. Tübingen: Stauffenburg.

Steube, Anita (1994). Syntaktische und semantische Eigenschaften sekundärer Prädikationen. In: Zur Satzwertigkeit von Infinitiven und Small Clauses, Anita Steube \& Gerhild Zybatow (Hgg.), 243-263. Tübingen: Niemeyer.

Thielmann, P. (1885). Habere mit dem Partizip Perfekt Passiv. Archiv für lateinische Lexikographie und Grammatik II: 372; 509.

Vennemann, Theo (1974). Topics, subjects and word order: From SXV to SVX via TVX. In Historical Linguistics. Vol.1: Syntax, Morphology, Internal and Comparative Reconstruction, John M. Anderson \& Charles Jones (eds.), 339-376. Amsterdam: North Holland.

Zimmermann, Rüdiger (1978). Structural change in the English auxiliary system: On the replacement of be by have. Folia Linguistica 6: 107-117. 